\title{
Chiral effective theory of strong interactions.
}

\author{
B.L.Ioffe \\ Institute of Theoretical and Experimental Physics \\ B.Cheremushkinskaya 25, 117218 Moscow,Russia
}

\begin{abstract}
The review of chiral effective theory (CET) is given. CET is based on quantum chromodynamics and describes the processes of strong interaction at low energies. It is proved, that CET comes as a consequence of the spontaneous violation of chiral symmetry in QCD - the appearance of chiral symmetry violating vacuum condensates. The Goldstone theorem for the case of QCD is proved and the existence of the octet of massless Goldstone bosons $(\pi, K, \eta)$ is demonstrated in the limit of massless $u, d, s$ quarks (or the triplet of massless pions in the limit $m_{u}, m_{d} \rightarrow 0$ ). It is shown, that the same phenomenon - the appearance of quark condensate in QCD - which causes the Goldstone bosons, results in appearance of violating chiral symmetry massive baryons. The general form of CET Lagrangian is derived. Few examples of higher order corrections to tree diagrams in CET are given. The WessZumino term (of order $p^{4}$ term in CET Lagrangian) is presented. Low energy sum rules are presented. QCD and CET at finite temperature are discussed. In the framework of CET the $T^{2}$ correction to quark condensate in QCD at finite temperature $T$ is calculated and the results of higher order temperature corrections are demonstrated. These results indicate on phase transition in QCD at $T \simeq 150-$ $200 \mathrm{MeV}$. The mixing of current correlators in order $T^{2}$ is proved.
\end{abstract}

PACS numbers: $12.38,12.39 . \mathrm{F}, 11.30 . \mathrm{R}$

Review submitted for publication to "Uspekhi Fiz. Nayk" 


\section{Contents}

1. Introduction.

2. The masses of light quarks.

3. Spontaneous violation of chiral symmetry. Quark condensate.

4. Goldstone theorem.

5. Nucleon mass and quark condensate.

6. Chiral effective theory at low energies.

7. Low energy sum rules in CET.

8. QCD and CET at finite temperature.

9. Conclusion.

10. References.

e-mail: ioffe@vitep5.itep.ru 


\section{Introduction}

It is generally accepted - and this fact is undisputable now - that the true theory of strong interactions is quantum chromodynamics (QCD), nonabelian gauge theory of interacting quarks and gluons. QCD possesses a striking property of asymptotic freedom: the coupling constant $\alpha_{s}\left(Q^{2}\right)$ decreases logarithmically as a function of momentum transfer square $Q^{2}$ at large $Q^{2}: \alpha_{s}\left(Q^{2}\right) \sim 1 / \ln Q^{2}$ at $Q^{2} \rightarrow \infty$ (or, what is equivalent, $\alpha_{s}$ decreases at small distances, $\left.\alpha_{s}(r) \sim 1 / \ln r\right)$. This property of QCD allows one to perform reliable theoretical calculation of the processes, proceeding at high momentum transfers (at small distances) by using perturbation theory. However, the same property of the theory involves the increase (in the framework of perturbative theory an unlimited ones) of the running coupling constant in QCD at small momentum transfer, i.e. at large distances. Physically, such growth is natural and, even more, it is needed, otherwise the theory would not be a theory of strong interactions. QCD possesses also the other remarkable property, the property of confinement: quarks and gluons cannot leave the region of their strong interaction and cannot be observed as real physical objects. Physical objects, observed experimentally at large distances, are hadrons-mesons and baryons. These two circumstances - the growth of coupling constant and the phenomenon of confinement, do not make it possible as a rule, to predict theoretically in QCD the processes at low energies and the properties of physical hadrons. (Some exceptions from this rule are low energy theorems, proved in QCD, and, especially the powerful QCD sum rule method, which, although, is starting from small distances, but allows one in many cases to go to rather large ones and to describe the properties of hadrons. The other exceptions are the numerical calculations on lattices.)

However, it became possible to construct the QCD based effective theory, which describes the processes of strong interaction at low energies. The small parameters in the theory are the momenta of interacting particles, more exactly, their ratios to the characteristic hadronic mass scale $M \sim 0.5-1 \mathrm{GeV}, p_{i} / M \ll 1$. The theory is constructed as a series in the powers of $p_{i} / M$ and is an effective theory, i.e., when going to the next order terms in $p_{i} / M$, in the Lagrangian one must take into account, additional terms, characterized by new parameters. The appearance of effective theory is connected with one more specific property of QCD - the spontaneous breaking of chiral symmetry. The masses of light $u, d, s$ quarks which enter the QCD Lagrangian, especially the masses of $u$ and $d$ quarks, from which the usual (nonstrange) hadrons are built, are very small as composed with characteristic mass scale $m_{u}, m_{d}<10 \mathrm{MeV}$. Since in QCD the quark interaction proceeds through the exchange of vector gluonic field, then, if light quark masses are neglected, QCD Lagrangian (its light quark part) is chirally symmetric, i.e. not only vector, but also axial currents are conserved and the left and right chirality quark fields are not interacting with one another. This chiral symmetry is not realized in the spectrum of hadrons and their low energy interactions. Indeed, in chirally symmetrical theory the fermion states must be either the massless or degenerate in parity. It is evident, that the baryons (particularly, the nucleon) do not possess such properties. This means, that the chiral symmetry of QCD Lagrangian is not realized on the spectrum of physical states and is spontaneously broken. According to Goldstone theorem spontaneous breaking of symmetry leads to appearance of massless particles in the spectrum of physical 
states - the Goldstone bosons. In QCD Goldstone bosons may be indentified with the triplet of $\pi$-mesons in the limit $m_{u}, m_{d} \rightarrow 0, m_{s} \neq 0(S U(2)$-symmetry) and the octet of pseudoscalar mesons $(\pi, K, \eta)$ in the limit $m_{u}, m_{d}, m_{s} \rightarrow 0$ (SU(3)-symmetry). The local $S U(2)_{V} \times S U(2)_{A}$ symmetry (here $V$ and $A$ mean vector and axial currents, $u$ and $d$ quarks are considered as massless) or $S U(3)_{V} \times S U(3)_{A}$ symmetry (if $m_{s}$ is also neglected) of hadronic strong interaction and the existence of massless Goldstone bosons allows one to construct the effective chiral theory of Goldstone bosons and their interactions with baryons, which is valid at small particle momenta.

In the initial version, before QCD, this approach was called the theory of partial conservation of axial current (PCAC). The Lagrangian of the theory represented the nonlinear interaction of pions with themselves and with nucleons and corresponded to the first term in the expansion in powers of momenta in modern chiral effective theory. (The review of the PCAC theory at this stage was done in [1]). When QCD had been created, it was proved, that the appearance of Goldstone bosons is a consequence of spontaneous breaking of chiral symmetry in QCD vacuum and it is tightly connected with the existence of vacuum condensates, violating the chiral symmetry. It had been also established, that baryon masses are expressed through the same vacuum condensates. Therefore, basing on QCD, the mutual interconnection of all set of phenomena under consideration was found. It was possible to formulate the chiral effective theory (CET) of hadrons as a succesive expansion in powers of particle momenta and quark (or Goldstone bosons) masses not only in tree approximation, as in PCAC, but also with account of loops. (CET is often called chiral perturbation theory - ChPT.)

In this review the foundations, basic ideas and concepts of CET are considered as well as their connection with QCD. The main attention is paid to the general properties of pion interactions. For pion-nucleon interaction only the general form of Lagrangian is presented. The physical effects are considered as illustrative examples in nonsystematical way. In fact in CET a lot of such effects was calculated (particularly, for meson-baryon interactions, meson and baryon formfactors etc.) They are very interesting for specialists, but their inclusion into review would increase its space drastically. The comparison of the theory with experiment almost will not be discussed. Such discussion could be a subject of a separate review.

\section{The masses of the light quarks}

In what follows $u, d, s$ quarks will be called as "light quarks" and all other quarks as "heavy quarks". The reason is that the masses of the light quarks are small compared with the characteristic mass of strong interaction $M \sim 0.5-1.0 \mathrm{GeV}$ or $m_{\rho}$. This statement is a consequence of the whole set of facts confirming that the symmetry of strong interaction is $S U(3)_{L} \times S U(3)_{R} \times U(1)$. Here the group generators are the charges corresponding to the left $(V-A)$ and right $(V+A)$ light quark chiral currents and $U(1)$ corresponds to the baryonic charge current. The experiment shows that the accuracy of $S U(3)_{L} \times S U(3)_{R}$ symmetry is of the same order as the accuracy of the $S U(3)$ symmetry: the small parameter characterizing the chiral symmetry violation in strong interactions is generally of order $\sim 1 / 5-1 / 10$. 
The approximate validity of the chiral symmetry means that not only divergences of the vector currents $\partial_{\mu} j_{\mu}^{q}$ are zero or small, but also of the axial ones $\partial_{\mu} j_{\mu 5}^{q}$. (Here $q=u, d, s$. This statement refers to nonsinglet in flavor axial currents. The divergence of singlet axial current is determined by the anomaly and is nonzero even for massless quarks - the discussion of this problem is outside the scope of this review). The divergences of nonsinglet axial currents in QCD are proportional to quark masses. Therefore the existence of the chiral symmetry can be understood if the quark masses are small [2, 3]. However, the baryon masses are by no means small: the chiral symmetry is not realized on the hadronic mass spectrum in a trivial way by vanishing of all the fermion masses. This means that the chiral symmetry is broken spontaneously by the physical states spectrum. According to the Goldstone theorem such a symmetry breaking results in appearance of massless particles - Goldstone bosons. In the case considered these Goldstone bosons must belong to a pseudoscalar octet. They are massless if quark masses are put to zero. The nonvanishing quark masses realize the explicit violation of the chiral symmetry and provide the masses of the pseudoscalar meson octet. For this reason the pseudoscalar meson octet (often called the octet of the Goldstone bosons) plays a special role in QCD.

Heavy quarks are decoupled in the low energy domain (this statement is called the Appelquist-Carazzone theorem) [4]. We ignore them in this Chapter where QCD at low energies is considered. The QCD Hamiltonian can be split into two pieces

$$
H=H_{0}+H_{1}
$$

where

$$
H_{1}=\int d^{3} x\left(m_{u} \bar{u} u+m_{d} \bar{d} d+m_{s} \bar{s} s\right)
$$

Evidently, because of vector gluon-quark interaction the first term in Hamiltonian $-H_{0}$ is $S U(3)_{L} \times S U(3)_{R}$ invariant and the only source of $S U(3)_{L} \times S U(3)_{R}$ violation is $H_{1}$. The quark masses $m_{q}, q=u, d, s$ in (2) are not renormalization invariant: they are scale dependent. It is possible to write

$$
m_{q}(M)=Z_{q}(M / \mu) m_{q}(\mu)
$$

where $M$ characterizes the scale, $\mu$ is some fixed normalization point and $Z_{q}(M / \mu)$ are renormalization factors. If the light quark masses are small and can be neglected, the renormalization factors are flavor-independent and the ratios

$$
\frac{m_{q 1}(M)}{m_{q 2}(M)}=\frac{m_{q 1}(\mu)}{m_{q 2}(\mu)}
$$

are scale-independent and have definite physical meaning. (This relation takes place if $M$ is higher than the Goldstone mass $m_{k}$ : its validity in the domain $M \sim m_{k}$ may lead to some doubts).

In order to find the ratios $m_{u} / m_{d}$ and $m_{s} / m_{d}$ consider the axial currents

$$
\begin{gathered}
j_{\mu 5}^{-}=\bar{d} \gamma_{\mu} \gamma_{5} u \\
j_{\mu 5}^{3}=\left[\bar{u} \gamma_{\mu} \gamma_{5} u-\bar{d} \gamma_{\mu} \gamma_{5} d\right] / \sqrt{2}
\end{gathered}
$$




$$
j_{\mu 5}^{s-}=\bar{s} \gamma_{\mu} \gamma_{5} u, \quad j_{\mu 5}^{s 0}=\bar{s} \gamma_{\mu} \gamma_{5} d
$$

and their matrix elements between vacuum and $\pi$ or $K$ meson states.

$$
\begin{aligned}
\left\langle 0\left|j_{\mu 5}^{-}\right| \pi^{+}\right\rangle & =i f_{\pi^{+}} p_{\mu} \\
\left\langle 0\left|j_{\mu 5}^{3}\right| \pi^{0}\right\rangle & =i f_{\pi^{0}} p_{\mu} \\
\left\langle 0\left|j_{\mu 5}^{s-}\right| K^{+}\right\rangle & =i f_{K^{+}} p_{\mu} \\
\left\langle\left|j_{\mu 5}^{s 0}\right| K^{0}\right\rangle & =i f_{K^{0}} p_{\mu}
\end{aligned}
$$

where $p_{\mu}$ are $\pi$ or $K$ momenta. In the limit of strict $S U(3)$ symmetry all constants in the rhs of (7) are equal: $f_{\pi^{+}}=f_{\pi^{0}}=f_{K^{+}}=f_{K^{0}}, S U(2)$ - isotopical symmetry results to equalities $f_{\pi^{+}}=f_{\pi^{0}}, f_{K^{+}}=f_{K^{0}}$. The constants $f_{\pi} \equiv f_{\pi}$ and $f_{K^{+}} \equiv f_{K}$ have the meaning of coupling constants in the decays $\pi^{+} \rightarrow \mu^{+} \nu$ and $K^{+} \rightarrow \mu^{+} \nu$. Experimentally they are equal to $f_{\pi}=131 \mathrm{MeV}, f_{K}=160 \mathrm{MeV}$. The ratio $f_{K} / f_{\pi}=1.22$ characterizes the accuracy of $S U(3)$ symmetry. Multiply (7) by $p_{\mu}$. Using the equality for the divergence of axial current following from QCD Lagrangian

$$
\partial_{\mu}\left[\bar{q}_{1}(x) \gamma_{\mu} \gamma_{5} q_{2}(x)\right]=i\left(m_{q_{1}}+m_{q_{2}}\right) \bar{q}_{1}(x) \gamma_{5} q_{2}(x)
$$

we get

$$
\begin{gathered}
i\left(m_{u}+m_{d}\right)\left\langle 0\left|\bar{d} \gamma_{5} u\right| \pi^{+}\right\rangle=f_{\pi^{+}} m_{\pi^{+}}^{2} \\
(i / \sqrt{2})\left[\left(m_{u}+m_{d}\right)\left\langle 0\left|\bar{u} \gamma_{5} u-\bar{d} \gamma_{5} d\right| \pi^{0}\right\rangle+\left(m_{u}-m_{d}\right)\left\langle 0\left|\bar{u} \gamma_{5} u+\bar{d} \gamma_{5} d\right| \pi^{0}\right\rangle\right]=f_{\pi^{0}} m_{\pi^{0}}^{2} \\
i\left(m_{s}+m_{u}\right)\left\langle 0\left|\bar{s} \gamma_{5} u\right| K^{+}\right\rangle=f_{K^{+}} m_{K^{+}}^{2} \\
i\left(m_{s}+m_{d}\right)\left\langle 0\left|\bar{s} \gamma_{5} d\right| K^{0}\right\rangle=f_{K^{0}} m_{K^{0}}^{2}
\end{gathered}
$$

Neglect electromagnetic (and weak) interaction and assume that isotopic invariance may be used for the matrix elements in the lhs of (9). Then

$$
\begin{gathered}
\left\langle 0\left|\bar{u} \gamma_{5} u+\bar{d} \gamma_{5} d\right| \pi^{0}\right\rangle=0 \\
\left\langle 0\left|\bar{d} \gamma_{5} u\right| \pi^{+}\right\rangle=\frac{1}{\sqrt{2}}\left\langle 0\left|\bar{u} \gamma_{5} u-\bar{d} \gamma_{5} d\right| \pi^{0}\right\rangle
\end{gathered}
$$

and, as follows from (9), $\pi^{ \pm}$and $\pi^{0}$ masses are equal in this approximation even when $m_{u} \neq m_{d}$. Hence the experimentally observed mass difference $\Delta m_{\pi}=m_{\pi^{+}}-m_{\pi^{0}}=$ 4.6 $\mathrm{MeV}$ is caused by the electromagnetic interaction only. The sign of the $K$-meson mass difference $\Delta m_{K}=m_{K^{+}}-m_{K^{0}}=-4.0 \mathrm{MeV}$ is opposite to that of the pion ones. The electromagnetic kaon and pion mass differences in QCD or in the quark model are determined by the same diagrams, and must, at least, to be of the same sign. This means, in accord with (9), that $m_{d}>m_{u}$.

Assuming the $S U(3)$ invariance of matrix elements in (9) and using simple algebra, it is easy to get from (9) and (10)

$$
\frac{m_{u}}{m_{d}}=\frac{\bar{m}_{\pi}^{2}-\left(\bar{m}_{K^{0}}^{2}-\bar{m}_{K^{+}}^{2}\right)}{\bar{m}_{\pi}^{2}+\left(\bar{m}_{K^{0}}^{2}-\bar{m}_{K^{+}}^{2}\right)}
$$




$$
\frac{m_{s}}{m_{d}}=\frac{\bar{m}_{K^{0}}^{2}+\bar{m}_{K^{+}}^{2}-\bar{m}_{\pi}^{2}}{\bar{m}_{K^{0}}^{2}-\bar{m}_{K^{+}}^{2}+\bar{m}_{\pi}^{2}}
$$

The bars in (11) mean, that the pion and kaon masses here are not the physical ones, but the masses in the limit, when the electromagnetic interaction is switched off. In order to relate $\bar{m}_{\pi}^{2}, \bar{m}_{K}^{2}$ to physical masses, let us use again the $S U(3)$ symmetry. In the $S U(3)$ symmetry the photon is $U$-scalar and $\pi^{+}$and $K^{+}$belong to the $U$ doublet. Therefore, the electromagnetic corrections to $m_{\pi^{+}}^{2}$ and $m_{K^{+}}^{2}$ are equal

$$
\left(\delta m_{\pi^{+}}^{2}\right)_{e l}=\left(\delta m_{K^{+}}^{2}\right)_{e l}
$$

It can be shown also, that in the limit $m_{\pi}^{2}, m_{K}^{2} \rightarrow 0$, the electromagnetic corrections to the $\pi^{0}$ and $K^{0}$ masses tend to zero,

$$
\left(\delta m_{\pi^{0}}^{2}\right)_{e l}=\left(\delta m_{K^{0}}^{2}\right)_{e l}=0
$$

Eq.'s (12), (13) may be rewritten in the form of the Dashen relation [5]

$$
\left(m_{\pi^{+}}^{2}-m_{\pi^{0}}^{2}\right)_{e l}=\left(m_{K^{+}}^{2}-m_{K^{0}}^{2}\right)_{e l}
$$

From (13), (14) we have

$$
\begin{gathered}
\bar{m}_{\pi}^{2}=m_{\pi^{0}}^{2} \\
\bar{m}_{K^{+}}^{2}-\bar{m}_{K^{0}}^{2}=m_{K^{+}}^{2}-m_{K^{0}}^{2}-\left(m_{\pi^{+}}^{2}-m_{\pi^{0}}^{2}\right)
\end{gathered}
$$

The substitution of (15) into (11) leads to:

$$
\begin{gathered}
\frac{m_{u}}{m_{d}}=\frac{2 m_{\pi^{0}}^{2}-m_{\pi^{+}}^{2}-\left(m_{K^{0}}^{2}-m_{K^{+}}^{2}\right)}{m_{K^{0}}^{2}-m_{K^{+}}^{2}+m_{\pi^{+}}^{2}} \\
\frac{m_{s}}{m_{d}}=\frac{m_{K^{0}}^{2}+m_{K^{+}}^{2}-m_{\pi^{0}}^{2}}{m_{K^{0}}^{2}-m_{K^{+}}^{2}+m_{\pi^{+}}^{2}}
\end{gathered}
$$

Numerically, this gives $[6,7]$

$$
\frac{m_{u}}{m_{d}}=0.56, \quad \frac{m_{s}}{m_{d}}=20.1
$$

A strong violation of isotopic invariance, as well as large difference between $u, d$ and $s$ quark masses, i.e. the violation of $S U(3)$ flavor symmetry, is evident from (17). (A more detailed analysis shows, that the results (17) only slightly depend on the assumption of the $S U(3)$ symmetry of the corresponding matrix elements used in their derivation.)This seems to be in contradiction with the well established isospin symmetry of strong interaction, as well as with the approximate $S U(3)$ symmetry. The resolution of this puzzle is that the quark masses are small: the parameter characterizing isospin violation is $\left(m_{d}-m_{u}\right) / M$ and the parameter characterizing the $S U(3)$ symmetry violation is $m_{s} / M$.

Estimation of the absolute value of the quark masses can be obtained in the following way. Suppose that the hadrons which contain strange quarks and which belong to a given unitary multiplet are heavier only because of the strange quark mass. Then from 
consideration of mass splittings in the baryon octet one can found that $m_{s} \approx 150 \mathrm{MeV}$ at a scale of about $1 \mathrm{GeV}$. From (17) it then follows

$$
m_{u}=4.2 \mathrm{MeV}, \quad m_{d}=7.5 \mathrm{MeV}, \quad m_{s}=150 \mathrm{MeV}
$$

at $1 \mathrm{GeV}$. Taking these values, one may expect that isospin violation could be of order $\left(m_{d}-m_{u}\right) / M \sim 10^{-2}$, i.e. of the same order as arising from electromagnetic interaction. The order of the $S U(3)$ symmetry violation is $\sim m_{s} / M \sim 1 / 5$. The large $m_{s} / m_{d}$ ratio explains the large mass splitting in the pseudoscalar meson octet. Assuming $\mathrm{SU}(3)$ symmetry of the matrix elements in (9), we have

$$
\frac{m_{K^{+}}^{2}}{m_{\pi^{+}}^{2}}=\frac{m_{s}+m_{u}}{m_{d}+m_{u}}=13
$$

in agreement with the experiment.

An important consequence of eq.'s (9) is that in QCD the mass squares of the pseudoscalar meson octet $m_{\pi}^{2}, m_{K}^{2}, m_{\eta}^{2}$ are proportional to the quark masses and vanish when $m_{q}$ tend to zero: in this limit the octet of pseudoscalar mesons becomes massless.

\section{Spontaneous violation of chiral symmetry}

\section{Quark condensate}

As has been already mentioned, the large baryon masses indicate that chiral symmetry in QCD is broken spontaneously. Indeed, let us consider any process with participation of a polarized baryon, e.g., any hadron-proton scattering on a longitudinally polarized proton at energies of order $1 \mathrm{GeV}$. We can treat the initial state of a polarized proton as a state with some fixed quark helicities. Due to the chiral symmetry the helicities are conserved in the course of collision. Therefore, we could expect that proton longitudinal polarization will not change in the collision. However, it is well known - and this is a direct consequence of the Dirac equation for proton - that the proton mass results in proton helicity flip with not a small probability in such a process. This simple fact - the existence of the proton mass - clearly demonstrates the violation of the chiral symmetry in strong interactions at low energies. 1

In all known examples of the field theories the spontaneous violation of global symmetry manifests itself in the modification of the properties of the ground state - the vacuum. Let us show that such phenomenon takes place also in QCD.

Consider the matrix element

$$
i q_{\mu}\left(m_{u}+m_{d}\right) \int d^{4} x e^{i q x}\left\langle 0\left|T\left\{j_{\mu 5}^{-}(x), \bar{u}(0) \gamma_{5} d(0)\right\}\right| 0\right\rangle
$$

in the limit of massless $u$ and $d$ quarks (except for the overall factor $m_{u}+m_{d}$ ). Put $q_{\mu}$ inside the integral, integrate in parts and use the conservation of the axial current. Then only the term with the equal time commutator will remain

\footnotetext{
${ }^{1}$ In principle, the chiral symmetry in baryonic states could be realized in a way that all baryonic states would be degenerated in parity with a splitting of order of $m_{u}+m_{d}$. This is evidently not the case.
} 


$$
\begin{gathered}
-\left(m_{u}+m_{d}\right) \int d^{4} x e^{i q x}\left\langle 0\left|\delta\left(x_{0}\right)\left[j_{05}^{-}(x), \quad \bar{u}(0) \gamma_{5} d(0)\right]\right| 0\right\rangle= \\
=\left(m_{u}+m_{d}\right)\langle 0|\bar{u} u+\bar{d} d| 0\rangle
\end{gathered}
$$

Let us go now to the limit $q_{\mu} \rightarrow 0$ in (20) and perform the summation over all intermediate states. The nonvanishing contribution comes only from one pion intermediate state, since in this approximation the pion should be considered as massless. This contribution is equal to

$$
q_{\mu}\left\langle 0\left|j_{\mu 5}^{-}\right| \pi^{+}\right\rangle \frac{-1}{q^{2}}\left\langle\pi^{+}\left|\left(m_{u}+m_{d}\right) \bar{u} \gamma_{5} d\right| 0\right\rangle=-f_{\pi}^{2} m_{\pi}^{2},
$$

where (7) and (9) where substituted when going to the right hand side. Putting (22) in the left hand side of (21) we get

$$
\langle 0|\bar{q} q| 0\rangle=-\frac{1}{2} \frac{m_{\pi}^{2} f_{\pi}^{2}}{m_{u}+m_{d}},
$$

where $q=u$ or $d$ and $\mathrm{SU}(2)$ invariance of QCD vacuum were used. Eq.(23) is called Gell-Mann-Oakes-Renner relation [8]. It can be also derived in an other way. Assume the quark masses to be nonzero, but small. Then the pion is massive and (20) tends to zero in the limit $q_{\mu} \rightarrow 0$. However, when we put $q_{\mu}$ inside the integral, in addition to the equal time commutator term (21), the term with the divergence of the axial current will appear. The account of this term, saturated by one pion intermediate state, results in the same eq.(23). Numerically, with the quark mass values (18) we have

$$
\langle 0|\bar{q} q| 0\rangle=-(240 \mathrm{MeV})^{3}
$$

As follows from (23), the product $\left(m_{u}+m_{d}\right)\langle 0|\bar{q} q| 0\rangle$ is scale independent, while $\langle 0|\bar{q} q| 0\rangle$ depends on the scale and the numerical value (24) refers to $1 \mathrm{GeV}$. The quantity $\langle 0|\bar{q} q| 0\rangle$, called vacuum quark condensate can be also represented as

$$
\langle 0|\bar{q} q| 0\rangle=\left\langle 0\left|\bar{q}_{L} q_{R}+\bar{q}_{R} q_{L}\right| 0\right\rangle
$$

where $q_{L}$ and $q_{R}$ are left and right quark fields $q_{L}=(1 / 2)\left(1+\gamma_{5}\right) q, \quad q_{R}=(1 / 2)\left(1-\gamma_{5}\right) q$. It is evident from (24) that quark condensate violates chiral invariance and its numerical value (24) has a characteristic hadronic scale. The chiral invariance is violated globally, because $\langle 0|\bar{q} q| 0\rangle$ is noninvariant under global transformations $q \rightarrow e^{i \alpha \gamma_{5}} q$ with a constant $\alpha$.

Surely, in perturbative QCD with massless quarks the quark condensate is zero in any order of perturbation theory. Therefore, the nonzero and non-small value of the quark condensate may arise only due to nonperturbative effects. The conclusion is, that the nonperturbative field fluctuations which violate chiral invariance of the Lagrangian, are present and essential in QCD. Quark condensate plays a special role because its lowest dimension, $\mathrm{d}=3$. 


\section{Goldstone theorem}

In Sec.2.2 we have presented two arguments in favor that chiral symmetry, approximately valid in QCD because of small $u, d, s$ quark masses, is spontaneously broken. These arguments were: the existence of large baryon masses and the appearance of violating chiral symmetry quark condensate. Let us go to the limit of massless $u, d, s$ quarks and show now that the direct consequence from each of these arguments is the appearance of massless pseudoscalar bosons in the hadronic spectrum.

Consider the matrix element of the axial current $j_{\mu 5}^{+}=\bar{u} \gamma_{\mu} \gamma_{5} d$ between the neutron and proton states. The general from of this matrix element is:

$$
\left\langle p\left|j_{\mu 5}^{+}\right| n\right\rangle=\bar{v}_{p}\left(p^{\prime}\right)\left[\gamma_{\mu} \gamma_{5} F_{1}\left(q^{2}\right)+q_{\mu} \gamma_{5} F_{2}\left(q^{2}\right)\right] v_{n}(p),
$$

where $p$ and $p^{\prime}$ are neutron and proton momenta, $q=p^{\prime}-p, \quad v_{p}\left(p^{\prime}\right), \quad v_{n}(p)$ are proton and neutron spinors and $F_{1}\left(q^{2}\right), F_{2}\left(q^{2}\right)$ are formfactors. Multiply (26) by $q_{\mu}$ and go to the limit $q^{2} \rightarrow 0$, but $q_{\mu} \neq 0$. After multiplication the lhs of (26) vanishes owing to axial current conservation. In the r.h.s using the Dirac equations for proton and neutron spinors, we have:

$$
\bar{v}_{p}\left(p^{\prime}\right)\left[2 m g_{A}+q^{2} F_{2}\left(q^{2}\right)\right] \gamma_{5} v_{n}(p),
$$

where $g_{A}=F_{1}(0)$ is the neutron $\beta$-decay coupling constant, $g_{A}=1.26$ and $m$ is the nucleon mass (assumed to be equal for proton and neutron). The only way to avoid the discrepancy with the vanishing lhs of (26) is to assume that $F_{2}\left(q^{2}\right)$ has a pole at $q^{2}=0$ :

$$
F_{2}\left(q^{2}\right)_{q^{2} \rightarrow 0}=-2 m g_{A} \frac{1}{q^{2}}
$$

The pole in $F_{2}\left(q^{2}\right)$ corresponds to appearance of a massless particle with pion quantum numbers. The matrix element in (26) has then the form (at small $q^{2}$ ):

$$
\left\langle p\left|j_{\mu 5}^{+}\right| n\right\rangle=g_{A} \bar{v}_{p}\left(p^{\prime}\right)\left(\delta_{\mu \nu}-\frac{q_{\mu} q_{\nu}}{q^{2}}\right) \gamma_{\nu} \gamma_{5} v_{n}(p),
$$

where conservation of the axial current is evident. The second term in the rhs of (29) can be described by the interaction of the axial current with the nucleon proceeding through intermediate pion, when the axial current creates virtual $\pi^{+}$and then $\pi^{+}$is absorbed by neutron (Fig.1). The low energy pion-nucleon interaction can be phenomenologically parametrized by the Lagrangian

$$
L_{\pi N N}=i g_{\pi N N} \bar{v}_{N} \gamma_{5} \tau^{a} v_{N} \varphi^{a}
$$

where $\tau^{a}$ are the isospin Pauli matrices and $g_{\pi N N}$ is the $\pi N N$ coupling constant, $g_{\pi N N}^{2} / 4 \pi \approx 14$. Using (17) and (30) the second term in (26) can be represented as

$$
-\sqrt{2} g_{\pi N N} f_{\pi} \bar{v}_{p} \gamma_{5} v_{n} \frac{q_{\mu}}{q^{2}}
$$




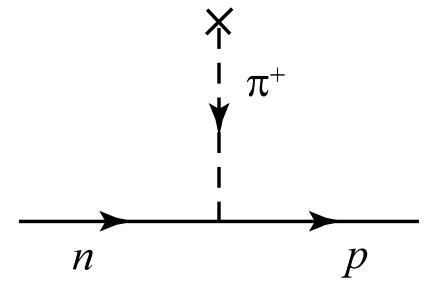

Figure 1. The diagram, describing the interaction of nucleon with axial current through intermediate pion: the solid lines correspond to nucleon, the dashed line - to pion, the cross means the interaction with external axial current.

The comparison with (28) gives the Goldberger-Treiman relation [9]

$$
g_{\pi N N} f_{\pi}=\sqrt{2} m g_{A}
$$

Experimentally, the Goldberger-Treiman relation is satisfied with a $5 \%$ accuracy, what strongly supports the hypothesis of spontaneous chiral symmetry violation in QCD. The main modification of (29) which arises from the nonvanishing pion mass is the replacement of the pion propagator: $q^{2} \rightarrow q^{2}-m_{\pi}^{2}$. Then the contribution of the second term vanishes at $q_{\mu} \rightarrow 0$ and becomes very small in the case of neutron $\beta$-decay.

Since the only assumption in the consideration above was the conservation of the axial current, this consideration can be generalized to any other component of the isospin 1 axial current, if $\mathrm{SU}(2)$ flavour symmetry is supposed, and to any octet axial current in the case of the $\mathrm{SU}(3)$ flavor symmetry. In the last case we come to the conclusion that the octet of pseudoscalar mesons is massless in the limit of massless $u, d, s$ quarks.

The massless bosons which arise through spontaneous symmetry breaking are called Goldstone bosons and the theorem which states their appearance is called Goldstone theorem [10] (see also [11]). The proof of the Goldstone theorem presented above was based on existence of massive baryons and on nonvanishing nucleon $\beta$-decay constant $g_{A}$. Before proceeding to another proof based on the existence of quark condensate in QCD, let us formulate some general features of spontaneously broken theories.

Let the Hamiltonian of the theory under consideration be invariant under some Lie group $G$, i.e., the group generators $Q_{i}$ to commute with the Hamiltonian

$$
\left[Q_{i}, H\right]=0, \quad i=1, \ldots n
$$

The symmetry is spontaneously broken if the ground state is not invariant under $G$ and a subset of $Q_{l}, l \leq m, 1 \leq m \leq n$ exists such that

$$
Q_{l}|0\rangle \neq 0
$$

Denote: $\left|B_{l}\right\rangle=Q_{l}|0\rangle$. As follows from (33)

$$
H\left|B_{l}\right\rangle=0
$$

- the states $\left|B_{l}\right\rangle$ have the same energy as vacuum. These states may be considered as 
massless bosons at rest - Goldstone bosons. If The generators $Q_{j}, j=m+1, \ldots n$ generate a subgroup $K \subset \mathrm{G}$, since from

$$
Q_{j}|0\rangle=0
$$

it follows

$$
\left[Q_{j}, Q_{j^{\prime}}\right]|0\rangle=0 \quad j, j^{\prime}=m+1, \ldots n
$$

In the case of QCD the group $G$ is $S U(3)_{L} \times S U(3)_{R}$, which is spontaneously broken to $S U(3)_{V}$ - the group, where generators are the octet of vector charges. $Q_{l}$ are the octet of axial charges and $\left|B_{l}\right\rangle$ are the octet of pseudoscalar mesons. (If only $u, d$ quarks are considered as massless, all said above may be repeated, but relative to $S U(2)_{L} \times S U(2)_{R}$ group).

Strictly speaking, the states $\left|B_{l}\right\rangle$ are not well defined, they have infinite norm. Indeed,

$$
\left\langle B_{l} \mid B_{l}\right\rangle=\left\langle 0\left|Q_{l} Q_{l}\right| 0\right\rangle=\int d^{3} x\left\langle 0\left|j_{l}(\mathbf{x}, t) Q_{l}(t)\right| 0\right\rangle,
$$

where $j_{l}(x)$ is the charge density operator corresponding to the generator $Q_{l}$.Extracting the $\mathbf{x}$-dependence of $j_{l}(\mathbf{x}, t)$ and using the fact that vacuum and intermediate states in (38) have zero momenta, we have

$$
\left\langle B_{l} \mid B_{l}\right\rangle=\int d^{3} x\left\langle 0\left|j_{l}(0, t) Q_{l}(t)\right| 0\right\rangle=V\left\langle\left|j_{l}(0, t), Q_{l}(t)\right| 0\right\rangle,
$$

where $\mathrm{V}$ is the total volume, $V \rightarrow \infty$. Physically, the infinite norm is well understood, since the massless Goldstone boson with zero momentum is distributed over the whole space. The prescription how to treat the problem is evident - to give a small mass to the boson. In what follows, when the commutators will be considered, the problem can be circumvented by performing first the commutation resulting in $\delta$-functions, and after integration over $d^{3} x$.

Let us demonstrate now, how this general theorem works in QCD in a explicit way. Go back to Eq.(21), which at $q=0$ may be rewritten as

$$
\left\langle 0\left|\left[Q_{5}^{-}, \bar{u} \gamma_{5} d\right]\right| 0\right\rangle=-\langle 0|\bar{u} u+\bar{d} d| 0\rangle
$$

where

$$
Q_{5}^{-}=\int d^{3} x j_{05}^{-}(x)
$$

is the axial charge generator. It is evident from (40), that $Q_{5}^{-}$does not annihilate vacuum, i.e. it belongs to the set of (34) generators. It is clear that the same property are inherent to all members of the octet of axial charges in SU(3) symmetry (or to members of isovector axial charges in SU(2) symmetry). Applying the general considerations of Goldstone, Salam and Weinberg [12 to our case, consider the vacuum commutator

$$
\left\langle 0\left|\left[j_{\mu 5}^{-}(x), \bar{u}(0) \gamma_{5} d(0)\right]\right| 0\right\rangle
$$

in coordinate space. Eq.(42) can be written via Lehmann-Källen representation

\footnotetext{
${ }^{2}$ The statement that $Q_{l}$ are generators of a continuous Lie group is essential - the theorem is not correct for discrete symmetry generators.
} 


$$
\left\langle 0\left|\left[j_{\mu 5}^{-}(x), \bar{u}(0) \gamma_{5} d(0)\right]\right| 0\right\rangle=\frac{\partial}{\partial x_{\mu}} \int d \kappa^{2} \Delta\left(x, \kappa^{2}\right) \rho^{-}\left(\kappa^{2}\right),
$$

where $\Delta\left(x, \kappa^{2}\right)$ is the Pauli-Jordan (causal) function for a scalar particle with mass $\kappa$

$$
\left(\partial_{\mu}^{2}+\kappa^{2}\right) \Delta\left(x, \kappa^{2}\right)=0
$$

and $\rho\left(\kappa^{2}\right)$ is the spectral function, defined by

$$
(2 \pi)^{-3} p_{\mu} \theta\left(p_{0}\right) \rho^{-}\left(p^{2}\right)=-\sum_{n} \delta^{4}\left(p-p_{n}\right)\left\langle 0\left|j_{\mu 5}^{-}(0)\right| n\right\rangle\left\langle n\left|\bar{u}(0) \gamma_{5} d(0)\right| 0\right\rangle
$$

The axial current conservation and (44) imply that

$$
\kappa^{2} \rho^{-}\left(\kappa^{2}\right)=0,
$$

hence

$$
\rho^{-}\left(\kappa^{2}\right)=N \delta\left(\kappa^{2}\right)
$$

The substitution of (47) into (43) gives

$$
\left\langle 0\left|\left[j_{\mu 5}^{-}(x), \bar{u}(0) \gamma_{5} d(0)\right]\right| 0\right\rangle=\frac{\partial}{\partial x_{\mu}} D(x) N,
$$

where $D(x)=\Delta(x, 0)$. Put $\mu=0, t=0$, integrate (48) over $d^{3} x$ and use the equality $\partial D(x) /\left.\partial t\right|_{t=0}=-\delta^{3}(x)$. The comparison of the result with (21) shows, that $N$ is proportional to quark condensate and nonzero. This means that the spectrum of physical states contains a massless Goldstone boson which gives a nonzero contribution to $\rho^{-}$. Its quantum numbers are those of $\pi^{+}$. It is easy to perform a similar consideration for other members of the pion multiplet in the case of $\mathrm{SU}(2)$ symmetry or for the pseudoscalar meson octet in the case of SU(3) symmetry. Obviously, the proof can be repeated for any other operator whose commutator with axial charges has nonvanishing vacuum average.

The presented above two proofs cannot be considered as a rigorous ones, like a mathematical theorem, where the presence of Goldstone bosons in QCD is proved starting from QCD Lagrangian and by use of the first principles of the theory. Indeed, in the first proof the existence of massive nucleon was taken as an experimental fact. In the second proof the appearance of nonvanishing quark condensate in QCD was exploited. The latter was proved (see eq.'s (20)-(22)) - basing on Ward identities, which, as was demonstrated, became selfconsistent only in the case of existence of massless pion. Therefore, these proofs may be treated as a convincing physical argumentation, but not a mathematical theorem (cf.[13]).

\section{Nucleon mass and quark condensate}

Let us show now, that the mentioned above two arguments in the favor of spontaneously broken chiral symmetry in QCD, namely, the existence of large baryon masses and the appearance of violating chiral symmetry quark condensate are in fact deeply interconnected. 


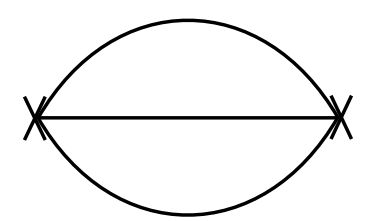

Figure 2. The bare loop diagram, contributing to chirality conserving function $f_{1}\left(p^{2}\right)$ : solid lines correspond to quark propagators, crosses mean the interaction with external currents.

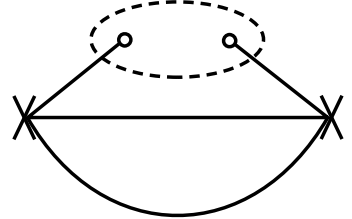

Figure 3. The diagram, corresponding to chirality violating dimension 3 operator (quark condensate). The dots, surrounded by circle mean quarks in the condensate phase. All other notation is the same as on Fig.2.

Demonstrate, that baryon masses arise just due to quark condensate. I will use the QCD sum rule method invented by Shifman, Vainstein and Zakharov [14], in its applications to baryons [15]. (For a review and collection of relevant original papers see [16]). The idea of the method is that at virtualities of order $Q^{2} \sim 1 \mathrm{GeV}^{2}$ the operator product expansion (OPE) may be used in consideration of hadronic vacuum correlators. In OPE the nonoperturbative effects reduce to appearance of vacuum condensates and condensates of the lowest dimension play the most important role. The perturbative terms are moderate and do not change the results in essential way, especially in the cases of chiral symmetry violation, where they can appear as corrections only.

For definiteness consider the proton mass calculation $[15,17]$. Introduce the polarization operator

$$
\Pi(p)=i \int d^{4} x e^{i p x}\langle 0|T \eta(x), \bar{\eta}(0)| 0\rangle
$$

where $\eta(x)$ is the quark current with proton quantum numbers and $p^{2}$ is chosen to be space-like, $p^{2}<0,\left|p^{2}\right| \sim 1 \mathrm{GeV}^{2}$. The current $\eta$ is the colourless product of three quark fields, $\eta=\varepsilon^{a b c} q^{a} q^{b} q^{c}, q=u, d$, the form of the current will be specialized below. The general structure of $\Pi(p)$ is

$$
\Pi(p)=\hat{p} f_{1}(p)+f_{2}(p)
$$

The first structure, proportional to $\hat{p}$ is conserving chirality, while the second is chirality violating.

For each of the functions $f_{i}\left(p^{2}\right), i=1,2$ the OPE can be written as:

$$
f_{i}\left(p^{2}\right)=\sum_{n} C_{n}^{(i)}\left(p^{2}\right)\left\langle 0\left|O_{n}^{(i)}\right| 0\right\rangle
$$

where $\left\langle 0\left|O_{n}^{(i)}\right| 0\right\rangle$ are vacuum expectation values (v.e.v) of various operators (vacuum condensates), $C_{n}^{(i)}$ are coefficient functions calculated in QCD. For the first, conserving chirality structure function $f_{i}\left(p^{2}\right)$ OPE starts from dimension zero $(d=0)$ unit operator. Its contribution is described by the diagram of Fig.2 and

$$
\hat{p} f_{1}\left(p^{2}\right)=C_{0} \hat{p} p^{4} \ln \left[\Lambda_{u}^{2} /\left(-p^{2}\right)\right]+\text { polynomial },
$$


where $C_{0}$ is a constant, $\Lambda_{u}$ is the ultraviolet cutoff. The OPE for chirality violating structure $f_{2}\left(p^{2}\right)$ starts from $d=3$ operator, and its contribution is represented by the diagram of Fig.3:

$$
f_{2}\left(p^{2}\right)=C_{1} p^{2}\langle 0|0 \bar{q} q| 0\rangle \ln \frac{\Lambda_{u}^{2}}{\left(-p^{2}\right)}+\text { polynomial }
$$

Let us for a moment restrict ourselves to this first order terms of OPE and neglect higher order terms (as well the perturbative corrections).

On the other hand, the polarizaion operator (49) may be expressed via the characteristics of physical states using the dispersion relations

$$
f_{i}(s)=\frac{1}{\pi} \int \frac{\operatorname{Im} f_{i}\left(s^{\prime}\right)}{s^{\prime}+s} d s^{\prime}+\text { polynomial, } \quad s=-p^{2}
$$

The proton contribution to $\operatorname{Im} \Pi(p)$ is equal to

$$
\operatorname{Im} \Pi(p)=\pi\langle 0|\eta| p\rangle\langle p|\bar{\eta}| 0\rangle \delta\left(p^{2}-m^{2}\right)=\pi \lambda_{N}^{2}(\hat{p}+m) \delta\left(p^{2}-m^{2}\right),
$$

where

$$
\langle 0|\eta| p\rangle=\lambda_{N} v(p)
$$

$\lambda_{N}$ is a constant, $v(p)$ is the proton spinor and $m$ is the proton mass. Still restricting ourselves to this rough approximation, we may take equal the calculated in QCD expression for $\Pi(p)$ (Eq.'s(52),(53)) to its phenomenological representation Eq.(55). The best way to get rid of unknown polynomial, is to apply to both sides of the equality the Borel(Laplace) transformation, defined as

$$
\mathcal{B}_{M^{2}} f(s)=\lim _{n \rightarrow \infty, s \rightarrow \infty, s / n=M^{2}=\text { Const }} \frac{s^{n+1}}{n !}\left(-\frac{d}{d s}\right)^{n} f(s)=\frac{1}{\pi} \int_{0}^{\infty} d s \operatorname{Im} f(s) e^{-s / M^{2}}
$$

if $f(s)$ is given by dispersion relation (54). Notice, that

$$
\mathcal{B}_{M^{2}} \frac{1}{s^{n}}=\frac{1}{(n-1) !\left(M^{2}\right)^{n-1}}
$$

Owing to the factor $1 /(n-1)$ ! in $(58)$ the Borel transformation suppresses the contributions of high order terms in OPE.

Specify now the quark current $\eta(x)$. It is clear from (55) that proton contribution will dominate in some region of the Borel parameter $M^{2} \sim m^{2}$ only in the case when both calculated in QCD functions $f_{1}$ and $f_{2}$ are of the same order. This requirement, together with the requirements of absence of derivatives and of renormcovariance fixes the form of current in unique way (for more details see $[15,18]$ ):

$$
\eta(x)=\varepsilon^{a b c}\left(u^{a} C \gamma_{\mu} u^{b}\right) \gamma_{\mu} \gamma_{5} d^{c}
$$

where $C$ is the charge conjugation matrix. With the current $\eta(x)(59)$ the calculations of the diagrams Fig.2 can be easily performed, the constants $C_{0}$ and $C_{1}$ are determined and 
after Borel transformation two equations (sum rules) arise (on the phenomenoloical sides of the sum rules only proton state is accounted)

$$
\begin{gathered}
M^{6}=\tilde{\lambda}_{N}^{2} e^{-m^{2} / M^{2}} \\
-2(2 \pi)^{2}\langle 0|\bar{q} q| 0\rangle M^{4}=m \tilde{\lambda}_{N}^{2} e^{-m^{2} / M^{2}} \\
\tilde{\lambda}_{N}^{2}=32 \pi^{4} \lambda_{N}^{2}
\end{gathered}
$$

It can be shown that this rough approximation is valid at $M \approx m$. Using this value of $M$ and dividing (60) on (61) we get a simple formula for proton mass [15]:

$$
m=\left[-2(2 \pi)^{2}\langle 0|\hat{q} q| 0\rangle\right]^{1 / 3}
$$

This formula demonstrates the fundamental fact, that the appearance of the proton mass is caused by spontaneous violation of chiral invariance: the presence of quark condensate. (Numerically, (62) gives the experimental value of proton mass with an accuracy better than 10\%).

A more refined treatment of the problem of the proton mass calculation was performed: high order terms of OPE were accounted, as well as excited states in the phenomenological sides of the sum rules and the stability of the Borel mass dependence was checked. In the same way, the hyperons, isobar and some resonances masses were calculated, all in a good agreement with experiment $[19,20,21]$. I will not dwell on these results. The main conclusion is: the origin of baryon masses is in spontaneous violation of chiral invariance the existence of quark condensate in QCD. Therefore, three phenomena: baryon masses, quark condensate and the appearance of Goldstone bosons are tightly connected.

\section{Chiral effective theory at low energies}

An effective chiral theory based on QCD and exploiting the existence and properties of the Goldstone bosons may be formulated. This theory is an effective low energy theory, what means that the theory is selfconsistent, but only in terms of expansion in powers of particle momenta (or in the derivatives of fields in the coordinate space). The Lagrangian is represented as a series of terms with increasing powers of momenta. The theory breaks down at sufficiently high momenta, the characteristic parameters are $\left|\mathbf{p}_{i}\right| / M$, where $\mathbf{p}_{i}$ are the spatial momenta of the Goldstone bosons entering the process under consideration and $M$ is the characteristic scale of strong interaction. (Since $\mathbf{p}_{i}$ depend on the reference frame, some care must be taken when choosing the most suitable frame in each particular case). The physical ground of the theory is the fact that in the limit of vanishing (or small enough) quark masses the spectrum of Goldstone bosons is separated by the gap from the spectrum of other hadrons. The chiral effective theory working in the domain $\left|\mathbf{p}_{i}\right| / M \ll 1$, is a selfconsistent theory and not a model. Such theory can be formulated basing on the $S U(2)_{L} \times S U(2)_{R}$ symmetry with pions as (quasi) Goldstone bosons. Then one may expect the accuracy of the theory to be of the order of the one of the isospin theory, i.e. of a few per cent. Or the theory may be based on the $S U(3)_{L} \times S U(3)_{R}$ 
symmetry with an octet of pseudoscalar bosons $\pi, K, \eta$ as (quasi) Goldstone bosons. In this case the accuracy of the theory is of order of violation of the $\mathrm{SU}(3)$ symmetry, i.e., of order $m_{s} / M \sim 10-20 \%$. For definiteness, the main part of this section deals with the case of $S U(2)_{L} \times S U(2)_{R}$.

The heuristic arguments for the formulation of the chiral theory are the following. In the limit of quark and pion mass going to zero (7) can be replaced by the field equation

$$
\begin{gathered}
j_{\mu 5}^{i}=-\left(f_{\pi} / \sqrt{2}\right) \partial_{\mu} \varphi_{\pi}^{i} \\
j_{\mu 5}^{i}=\bar{q} \gamma_{\mu} \gamma_{5}\left(\tau^{i} / 2\right) q, q=u, d
\end{gathered}
$$

where $\varphi_{\pi}^{i}$ is the pion field, $\tau^{i}$ are the Pauli matrices and $i=1,2,3$ is isospin index. (Normalization of the current $j_{\mu 5}^{i}$ is changed comparing with (7) in order to have the standard commutation relations of current algebra). Taking the divergence from (63) we have

$$
\partial_{\mu} j_{\mu 5}^{i}=\left(f_{\pi} / \sqrt{2}\right) m_{\pi}^{2} \varphi_{\pi}^{i}
$$

Eqs.(63), (65) are correct near the pion mass shell.

Since the pion state is separated by the gap from the other massive states in the channel with pion quantum numbers these equations can be treated as a field equations valid in the low energy region (usually they are called the equations of partial conservation of axial current PCAC).

The direct consequence of (65) is the Adler selfconsistency condition [22]. Consider the amplitude of the process $A \rightarrow B+\pi$, where A and $\mathrm{B}$ are arbitrary hadronic states in the limit of vanishing pion momentum $p$. The matrix element of this process can be written as

$$
M_{i}(2 \pi)^{4} \delta^{4}\left(p_{A}-p-p_{B}\right)=\int d^{4} x e^{i p x}\left(\partial_{\mu}^{2}+m_{\pi}^{2}\right)\left\langle B\left|\varphi_{\pi}^{i}\right| A\right\rangle
$$

The substitution of (65) gives

$$
M_{i}=\frac{i\left(p^{2}-m_{\pi}^{2}\right)}{\left(f_{\pi} / \sqrt{2}\right) m_{\pi}^{2}} p_{\mu}\left\langle B\left|j_{\mu 5}^{i}(0)\right| A\right\rangle
$$

Going to the limit $p_{\mu} \rightarrow 0$ we get

$$
M(A \rightarrow B \pi)_{p \rightarrow 0} \rightarrow 0
$$

what is the Adler condition. When deriving (68) it was implicitly assumed that the matrix element $\left\langle B\left|j_{\mu 5}^{i}\right| A\right\rangle$ does not contain pole terms, where the axial current interacts with an external line. Generally, the Adler theorem does not work in such cases.

The chiral theory is based on the following principles:

1. The pion field transforms under some representation of the group $G=S U(2)_{L} \times$ $S U(2)_{R}$.

2. The action is invariant under these transformations.

3. After breaking the transformations reduce to $S U(2)$ - the transformations, which are generated by the isovector vector current. 
4. In the lowest order the field equations (63), (65) are fulfilled.

The pion field may be represented by the $2 \times 2$ unitary matrix $U(x), U^{-1}=U^{+}(x)$, depending on $\varphi_{\pi}^{i}(x)$. The condition $\operatorname{det} U=1$ is imposed on $U(x)$. Therefore the number of degrees of freedom of matrix $U$ is equal to that of three pionic fields $\varphi_{\pi}^{i}(x)$. The transformation law under the group $G$ transformations is given by

$$
U^{\prime}(x)=V_{L} U(x) V_{R}^{+}
$$

where $V_{L}$ and $V_{R}$ are unitary matrices of $S U(3)_{L}$ and $S U(3)_{R}$ transformations. (69) satisfies the necessary condition, that after breaking, when $G$ reduces to $S U(2)$ and $V_{L}=$ $V_{R}=V$, the transformation law reduces to

$$
U^{\prime}=V U(x) V^{-1}
$$

- the transformation, induced by the vector current.

It can be shown that the general form of the lowest order effective Lagrangian, where only the terms up to $p^{2}$ are kept and the breaking arising from the pion mass is neglected, is: [23]-[26]

$$
L_{e f f}=k \operatorname{Tr}\left(\partial_{\mu} U \cdot \partial_{\mu} U^{+}\right),
$$

where $k$ is some constant.

The conserving vector and the axial currents (Noether currents), corresponding to Lagrangian (71) can be found by applying to (71) the transformations (69) with

$$
V_{L}=V_{R}=1+i \vec{\varepsilon} \vec{\tau} / 2
$$

in case of vector current and

$$
V_{L}=V_{R}^{+}=1+i \vec{\varepsilon} \vec{\tau} / 2,
$$

in case of axial current. (Here $\vec{\varepsilon}$ is an infinitesimal isovector). The results are:

$$
\begin{gathered}
j_{\mu}^{i}=i k \operatorname{Tr}\left(\tau_{i}\left[\partial_{\mu} U, U^{+}\right]\right) \\
j_{\mu 5}^{i}=i k \operatorname{Tr}\left(\tau_{i}\left\{\partial_{\mu} U, U^{+}\right\}\right)
\end{gathered}
$$

One may use various realizations of the matrix field $U(x)$ in terms of pionic fields $\varphi_{\pi}^{i}(x)$. All of them are equivalent and lead to the same physical consequences [27, 28]. Mathematically, this is provided by the statement that one realization differs from the other by a unitary (nonlinear) transformation (69). One of the useful realizations is

$$
U(x)=\exp \left(i \alpha \vec{\tau} \vec{\varphi}_{\pi}(x)\right),
$$

where $\alpha$ is a constant. Substitution of (75) into (71) and expansion in power of pionic field up to the 4-th power gives

$$
L_{e f f}=2 k \alpha^{2}\left(\partial_{\mu} \vec{\varphi}_{\pi}\right)^{2}+\frac{2}{3} k \alpha^{4}\left[\left(\vec{\varphi}_{\pi} \partial_{\mu} \vec{\varphi}_{\pi}\right)^{2}-\vec{\varphi}_{\pi}^{2} \cdot\left(\partial_{\mu} \vec{\varphi}_{\pi}\right)^{2}\right]+\ldots
$$


From the requirement that the first, kinetic energy, term in (76) has the standard from, we have

$$
k \alpha^{2}=\frac{1}{4}
$$

Substitution of (75) into (74) in the first nonvanishing order in pionic field and account of (77) results in:

$$
\begin{aligned}
& j_{\mu}^{i}=\varepsilon_{i k l} \varphi_{\pi}^{k} \frac{\partial \varphi_{\pi}^{l}}{\partial x_{\mu}} \\
& j_{\mu 5}^{i}=-2 \sqrt{k} \frac{\partial \varphi_{\pi}^{i}}{\partial x_{\mu}}
\end{aligned}
$$

The formula for the vector current - the first in eqs.(78) is the standard formula for the pion isovector current. The comparison of the second equation (78) with (63) finally fixes the constant $k$ and, because of (77), $\alpha$

$$
k=\frac{1}{8} f_{\pi}^{2}, \alpha=\frac{\sqrt{2}}{f_{\pi}}
$$

Therefore, the effective Lagrangian (71) as well as $U(x)$ are expressed through one parameter - the pion decay constant $f_{\pi}$, which plays the role of the coupling constant in the theory. From dimensional grounds it is then clear that the expansions in powers of the momenta or in powers of pionic field are in fact the expansions in $p^{2} / f_{\pi}^{2}$ and $\varphi^{2} / f_{\pi}^{2}$. Particularly, the expansion of the effective Lagrangian (76) takes the form

$$
L_{e f f}=\frac{1}{2}\left(\partial_{\mu} \vec{\varphi}_{\pi}\right)^{2}+\frac{1}{3} \frac{1}{f_{\pi}^{2}}\left[\left(\vec{\varphi}_{\pi} \partial_{\mu} \vec{\varphi}_{\pi}\right)^{2}-\vec{\varphi}_{\pi}^{2}\left(\partial_{\mu} \vec{\varphi}_{\tau}\right)^{2}\right]+\ldots
$$

Turn now to symmetry breaking term in the chiral effective theory Lagrangian. This term is proportional to the quark mass matrix

$$
\mathcal{M}=\left(\begin{array}{ll}
m_{u} & 0 \\
0 & m_{d}
\end{array}\right)
$$

In the QCD Lagrangian the corresponding term transforms under $S U(2)_{L} \times S U(2)_{R}$ transformations according to representation $\frac{1}{2}, \frac{1}{2}$. This statement may be transferred to chiral theory by the requirement that in chiral theory the mass matrix (81) transforms according to

$$
\mathcal{M}^{\prime}=V_{R} \mathcal{M} V_{L}^{+}
$$

The term in the Lagrangian linear in $\mathcal{M}$ and of the lowest (zero) order in pion momenta, invariant under $S U(2)_{L} \times S U(2)_{R}$ transformation has the form

$$
L^{\prime}=\frac{f_{\pi}^{2}}{4}\left\{B \operatorname{Tr}\left(\mathcal{M} U^{+}\right)+B^{*} \operatorname{Tr}(\mathcal{M U})\right\}
$$


where $B$ is a constant and the factor $f_{\pi}^{2}$ is introduced for convenience. Impose the requirement of T-invariance to the Lagrangian (83). The pion field is odd under $T\left(\varphi_{\pi}^{i}\right)=-\varphi_{\pi}^{i}$, so $T U=U^{+}$and, as a consequence, $B=B^{*}$ and

$$
L^{\prime}=\frac{f_{\pi}^{2}}{4} B \operatorname{Tr}\left[\mathcal{M}\left(U+U^{*}\right)\right]
$$

In the lowest orders of the expansion in pionic fields (84) reduces to

$$
L^{\prime}=\frac{1}{2} B\left(m_{u}+m_{d}\right)\left[f_{\pi}^{2}-\vec{\varphi}_{\pi}^{2}+\frac{1}{6 f_{\pi}^{2}}\left(\vec{\varphi}_{\pi}^{2}\right)^{2}\right]
$$

The first term in the square bracket gives a shift in vacuum energy resulting from symmetry breaking, the second corresponds to the pion mass term in the Lagrangian $-\left(m_{\pi}^{2} / 2\right) \vec{\varphi}_{\pi}^{2}$. With this identification we can determine the constant $B$ :

$$
B=\frac{m_{\pi}^{2}}{m_{u}+m_{d}}=-\frac{2}{f_{\pi}^{2}}\langle 0|\bar{q} q| 0\rangle,
$$

where the Gell-Mann-Oakes-Renner relation (23) was used. The relation (86) can be also obtained in an another way. We have from the QCD Lagrangian

$$
\frac{\partial}{\partial m_{u}}\langle 0|L| 0\rangle=-\langle 0|\bar{u} u| 0\rangle
$$

Differentiating (85) we get:

$$
\frac{1}{2} B f_{\pi}^{2}=-\langle 0|\bar{u} u| 0\rangle
$$

what coincides with (86).

As a simplest application of the effective Lagrangians (80), (85), calculate the pionpion scattering amplitude in the first order in $1 / f_{\pi}^{2}$. The results are [29]:

$$
T=\delta^{i k} \delta^{l m} A(s, t, u)+\delta^{i l} \delta^{k m} A(t, s, u)+\delta^{i m} \delta^{k l} A(u, t, s)
$$

where

$$
\begin{gathered}
A(s, t, u)=\frac{2}{f_{\pi}^{2}}\left(s-m_{\pi}^{2}\right) \\
s=\left(p_{1}+p_{2}\right)^{2}, t=\left(p_{1}-p_{3}\right)^{2}, u=\left(p_{1}-p_{4}\right)^{2}
\end{gathered}
$$

$p_{1}, p_{2}$ - are initial and $p_{3}, p_{4}$ are final pion momenta. The isospin indices $i, k$ refer to initial pions, $l, m$ - to final ones. For example, for the $\pi^{+} \pi^{0} \rightarrow \pi^{+} \pi^{0}$ scattering amplitude we get 29]

$$
T=\frac{2}{f_{\pi}^{2}}\left(t-m_{\pi}^{2}\right)
$$

where $T$ is related to the c.m. scattering amplitude $f_{\text {c.m. }}$. by 


$$
f_{c . m .}=\frac{1}{16 \pi} \frac{1}{E} T
$$

and $E$ is the energy of $\pi^{+}$in c.m.system.

The other, instead of (75), often used realization is

$$
U(x)=\frac{\sqrt{2}}{f_{\pi}}\left[\sigma(x)+i \vec{\tau} \vec{\varphi}_{\pi}(x)\right]
$$

supplemented by the constrain

$$
\sigma^{2}+\vec{\varphi}^{2}=\frac{1}{2} f_{\pi}^{2}
$$

It can be shown by direct calculations, that the expressions for effective Lagrangians up to $\varphi^{4}$ obtained in this realization coincide with (80), (85) on pion mass shell. In higher orders $\left(\varphi^{6}, \varphi^{8}\right.$ etc. $)$ the expressions for effective Lagrangians in these two realizations are different even on mass shell. But, according to general arguments by Coleman, Wess and Zumino [27], the physical amplitudes became to be equal after adding one-particle reducible tree diagrams. Since the $\mathrm{SU}(2)$ group is isomorphic to $O(3)$ the realization (94) is equivalent to the one, where the $O(4)$ real four-vector $U_{i}(x)$, which satisfies the constrain $U_{i} U_{i}^{T}=1, i=1,2,3,4$, is used instead of the $2 \times 2$ matrix $U(x)$ [25].

The chiral effective Lagrangian (71) is the leading term in the expansion in pion momenta. The next term of order of $p^{4}$ consistent with Lorentz and chiral invariance, parity and $G$-parity symmetry has the general form 25]

$$
L_{2, e f f}=l_{1}\left[\operatorname{Tr}\left(\partial_{\mu} U \partial_{\mu} U^{+}\right)\right]^{2}+l_{2} \operatorname{Tr}\left(\partial_{\mu} U \partial_{\nu} U^{+}\right) \operatorname{Tr}\left(\partial_{\mu} U \partial_{\nu} U^{+}\right)
$$

where $l_{1}$ and $l_{2}$ are constants. The term of the second order in quark masses is added to (96). If spacial momenta of pions in the process under consideration are close to zero $|\mathbf{p}| \ll m_{\pi}$, the contribution of this term is of the same order as (96), since $p^{2}=m_{\pi}^{2} \sim$ $\left(m_{u}+m_{d}\right)$. Its general form is [25]

$$
\begin{gathered}
L_{2, e f f}^{\prime}=l_{4} \operatorname{Tr}\left(\partial_{\mu} U \partial_{\mu} U^{+}\right) \operatorname{Tr}\left[\chi\left(U+U^{+}\right)\right]+l_{6}\left\{\operatorname{Tr}\left[\chi\left(U+U^{+}\right)\right]\right\}^{2} \\
+l_{7}\left\{\operatorname{Tr}\left[i \chi\left(U-U^{+}\right)\right]\right\}^{2}
\end{gathered}
$$

where

$$
\chi=2 B \mathcal{M}
$$

In order to perform the next to leading order calculations in chiral effective theory it is necessary, besides the (96), (97) contribution, to go beyond the tree approximation in the leading order Lagrangians and to calculate one-loop contributions arising from (71), (84). As can be seen, the parameter of the expansion is $\left(1 / \pi f_{\pi}\right)^{2} \sim(1 / 500 \mathrm{MeV})^{2}$ and, as a rule, small numerical coefficients also arise. Therefore, the n-loops contribution is suppressed compared by the leading order tree approximation by the factor $\left[p^{2} /\left(\pi f_{\pi}\right)^{2}\right]^{n}$. Loop integrals are divergent and require renormalization. Renormalization can be performed in an any scheme which preserves the symmetry of the theory. These can be dimensional 
regularization or a method where finite imaginary parts of the scattering amplitudes are calculated and the whole amplitudes are reconstructed by using dispersion relations (an example of such calculation is given below) or any others. The counter terms arising in loop calculations (pole contributions at $d \rightarrow 4$ in dimensional regularization or subtraction constants in the dispersion relation approach) are absorbed by the coupling constants of the next order effective Lagrangian, like $l_{1}$ and $l_{2}$ in (96). Theoretically unknown constants $l_{i}$ are determined by comparing with the experimental data.

As a result of loop calculations and of the account of higher order terms in the effective Lagrangian the coupling constant $f_{\pi}$ entering (71), (84) acquire some contributions and is no more equal to the physical pion decay constant defined by (84). For this reason the coupling constant $f_{\pi}$ in (71), (84) should be considered as a bare one, $f_{\pi}^{0}$ which will coincide with the physical $f_{\pi}$ after accounting of all higher order corrections. A similar statement refers to the connection between $m_{\pi}^{2}$ and $m_{u}+m_{d}$ (86). If $B$ is considered as a constant parameter of the theory, then the relation (86) is modified by high order terms. Particularly, in the next to leading order 30

$$
m_{\pi}^{2}=\tilde{m}_{\pi}^{2}\left[1+c(\mu) \frac{m_{\pi}^{2}}{f_{\pi}^{2}}+\frac{m_{\pi}^{2}}{16 \pi^{2} f_{\pi}^{2}} \ln \frac{m_{\pi}^{2}}{\mu^{2}}\right]
$$

where

$$
\tilde{m}_{\pi}^{2}=B\left(m_{u}+m_{d}\right),
$$

$\mu$ is the normalization point and $c(\mu)$ is the $\mu$-depending renormalized coupling constant expressed through $l_{i}$. (The total correction is $\mu$-independent). The appearance of the nonanalytic in $m_{\pi}^{2}$ (or $m_{q}$ ) term $\sim m_{\pi}^{2} l n m_{\pi}^{2}$ - the so called "chiral logarithm" - is a specific feature of the chiral perturbation theory. The origin of their appearance are infrared singularities of the corresponding loop integrals. $f_{\pi}$ also contains the chiral logarithm [30]:

$$
f_{\pi}=f_{\pi}^{0}\left[1+c_{1}(\mu) \frac{m_{\pi}^{2}}{f_{\pi}^{2}}-\frac{m_{\pi}^{2}}{8 \pi^{2} f_{\pi}^{2}} \ln \frac{m_{\pi}^{2}}{\mu^{2}}\right]
$$

Let us present two examples of loop calculations.

1. Find the nonanalytical, proportional to $\ln m_{\pi}^{2}$ correction to pion electric radius $[31,32]$.

The one-loop contribution to pion formfactor comes from $\pi \pi$ interaction term in the Lagrangian given by (80) and is equal to

$$
i \frac{1}{f_{\pi}^{2}} \int \frac{d^{4} k_{1} d^{4} k_{2}}{(2 \pi)^{4}} \delta\left(q+k_{1}-k_{2}\right)\left(k_{1}+k_{2}\right)_{\mu} \frac{1}{k_{1}^{2}-m_{\pi}^{2}} \frac{1}{k_{2}^{2}-m_{\pi}^{2}}\left(p_{1}+p_{2}\right)\left(k_{1}+k_{2}\right)
$$

Here $p_{1}$ and $p_{2}$ are the initial and final pion momenta $q$ is the momentum transfer, $q^{2}<0, p_{1}+q=p_{2}$. The integral in (102) can be calculated in the following way. Consider the integral

$$
i \int \frac{d^{4} k_{1} d^{4} k_{2}}{(2 \pi)^{4}}\left(k_{1}+k_{2}\right)_{\mu}\left(k_{1}+k_{2}\right)_{\nu} \frac{1}{k_{1}^{2}-m_{\pi}^{2}} \frac{1}{k_{2}^{2}-m_{\pi}^{2}} \delta^{4}\left(q+k_{1}-k_{2}\right)=
$$




$$
=A\left(q^{2}\right)\left(\delta_{\mu \nu} q^{2}-q_{m u} q_{\nu}\right)
$$

The form of the rhs of (103) follows from gauge invariance. Calculate the imaginary part of $A\left(q^{2}\right)$ at $q^{2}>0$. We have

$$
\begin{aligned}
\operatorname{ImA} A\left(q^{2}\right)\left(\delta_{\mu \nu} q^{2}-q_{\mu} q_{\nu}\right)= & -\frac{1}{8 \pi^{2}} \int d^{4} k(2 k-q)_{\mu}(2 k-q)_{\nu} \delta\left[(q-k)^{2}\right]= \\
& =\frac{1}{48 \pi}\left(q^{2} \delta_{\mu \nu}-q_{\mu} q_{\nu}\right)
\end{aligned}
$$

(The pion mass can be neglected in our approximation). $A\left(q^{2}\right)$ is determined by dispersion relation:

$$
A\left(q^{2}\right)=\frac{1}{\pi} \int_{4 m_{\pi}^{2}}^{M^{2}} \frac{d s}{s-q^{2}} \operatorname{Im} A\left(q^{2}\right)=\frac{1}{48 \pi^{2}} \ln \frac{M^{2}}{4 m_{\pi}^{2}-q^{2}}
$$

(The subtraction term is omitted, $M^{2}$ is a cutoff). Substitution of (103), (104) into (102) gives for the correction to the $\gamma \pi \pi$ vertex

$$
\left(p_{1}+p_{2}\right)_{\mu}\left[F\left(q^{2}\right)-1\right]=\left(p_{1}+p_{2}\right)_{\mu} \frac{q^{2}}{48 \pi^{2} f_{\pi}^{2}} \ln \frac{M^{2}}{4 m_{\pi}^{2}-q^{2}},
$$

where $F\left(q^{2}\right)$ is the pion formfactor. The pion electric radius is defined by

$$
r_{\pi}^{2}=6 \frac{d F\left(q^{2}\right)}{d q^{2}}
$$

and its nonanalytical in $m_{\pi}^{2}$ part is equal to

$$
r_{\pi}^{2}=-\frac{1}{8 \pi^{2} f_{\pi}^{2}} \ln m_{\pi}^{2}
$$

2. Quark condensate also become the nonanalytical, proportional to $m_{\pi}^{2} l n m_{\pi}^{2}$ correction [33]. Using (87) and (85) we get

$$
\langle 0|\bar{u} u| 0\rangle=-\frac{1}{2} f^{2} \pi B\left\langle 0\left|1-\frac{\varphi_{i}^{2}}{f_{\pi}^{2}}\right| 0\right\rangle
$$

The mean vacuum value of $\varphi_{i}^{2}$ is given by

$$
\begin{gathered}
\lim _{x \rightarrow 0}\left\langle 0\left|T \varphi_{i}(x), \varphi_{i}(0)\right| 0\right\rangle=\frac{3 i}{(2 \pi)^{4}} \lim _{x \rightarrow 0} \int d^{4} k \frac{e^{i k x}}{k^{2}-m_{\pi}^{2}}= \\
=A m_{\pi}^{2}+C m_{\pi}^{2} \ln m_{\pi}^{2}+\ldots
\end{gathered}
$$

In order to find $C$ differentiate (110) over $m_{\pi}^{2}$. We have

$$
\frac{3 i}{(2 \pi)^{4}} \int d^{4} k \frac{1}{\left(k^{2}-m_{\pi}^{2}\right)^{2}}=-\frac{3 \pi^{2}}{(2 \pi)^{4}} \ln \frac{M^{2}}{m_{\pi}^{2}}
$$

Substitution of (111) into (109) with the account of (86) gives 


$$
\langle 0|\bar{u} u| 0\rangle=\langle 0|\bar{u} u| 0\rangle_{0}\left(1+\frac{3 m_{\pi}^{2}}{16 \pi^{2} f_{\pi}^{2}} \ln \frac{M^{2}}{m_{\pi}^{2}}+A m_{\pi}^{2}\right)
$$

Generalization for three massless quark case, when $s$-quark is also considered as massless and the symmetry of the Lagrangian is $S U(3)_{L} \times S U(3)_{R}$ is straightforward. The matrix $U(x)$ is $3 \times 3$ unitary matrix, the leading order Lagrangian has the same forms (71), (84) with an evident difference that the quark mass matrix $\mathcal{M}$ is now $3 \times 3$ matrix. In the formulae for axial and vector currents (64), (74) $\tau_{i}$ should be substituted by the Gell-Mann matrices $\lambda_{n}, n=1, \ldots 8$ and the same substitution must be done in the exponential realization of $U(x)$ :

$$
U(x)=\exp \left(i \frac{\sqrt{2}}{f_{\pi}} \sum_{n} \lambda_{n} \varphi_{n}(x)\right)
$$

where $\varphi_{n}(x)$ is the octet of pseudoscalar mesonic fields. Because the algebra of $\lambda_{n}$ matrices differs from that of $\tau_{i}$ and, particularly, the anticommutator $\lambda_{n}, \lambda_{m}$ does not reduce to $\delta_{n m}$, the linear realization as simple as (94) is impossible in this case.

The symmetry breaking Lagrangian (84) in the order of $\varphi_{n}^{2}-$ the mass term in the pseudoscalar meson Lagrangian - is nondiagonal in mesonic fields: the effective Lagrangian contains the term proportional to $\left(m_{u}-m_{d}\right) A \varphi_{3} \varphi_{8}$. The presence of this term means that the eigenstates of the Hamiltonian, $\pi^{0}$ and $\eta$ mesons, are not eigenstates of $Q^{3}$ and $Q^{8}$ generators of $S U(3)_{V}$ : in $\eta$ there is an admixture of the isospin 1 state (the pion) and vice versa $[34,35]$.

In general we can write:

$$
H=\frac{1}{2} \tilde{m}_{\pi}^{2} \varphi_{3}^{2}+\frac{1}{3} \tilde{m}_{\eta}^{2} \varphi_{8}^{2}+A\left(m_{u}-m_{d}\right) \varphi_{3} \varphi_{8}+\text { kinetic terms }
$$

The physical $\pi$ and $\eta$ states arises after orthogonalization of the Hamiltonian (114)

$$
\begin{aligned}
& |\pi\rangle=\cos \theta\left|\varphi_{3}\right\rangle-\sin \theta\left|\varphi_{8}\right\rangle \\
& |\eta\rangle=\sin \theta\left|\varphi_{3}\right\rangle+\cos \theta\left|\varphi_{8}\right\rangle
\end{aligned}
$$

It can be shown $[33,34,26]$, that the constant $A$ in (114) is equal

$$
A=\frac{1}{\sqrt{3}} \frac{m_{\pi}^{2}}{m_{u}+m_{d}}
$$

and the mixing angle is given by (at small $\theta$ )

$$
\theta=\frac{1}{\sqrt{3}} \frac{m_{\pi}^{2}}{m_{\eta}^{2}-m_{\pi}^{2}} \frac{m_{u}-m_{d}}{m_{u}+m_{d}}
$$

This result is used in consideration of many problems, where isospin is violated, e.q. the decay rate $\psi^{\prime} \rightarrow J / \psi \pi^{0}$ [36], the amplitude of $\eta \rightarrow \pi^{+} \pi^{-} \pi^{0}$ decay. The violating isospin amplitude $\eta \rightarrow \pi^{+} \pi^{-} \pi^{0}$ is found to be [37,38] (in its derivation (Eq.(117)) was exploited): 


$$
T_{\eta \rightarrow \pi^{+} \pi^{-} \pi^{0}}=\frac{\sqrt{3}}{2 f_{\pi}^{2}} \frac{m_{u}-m_{d}}{m_{s}-\left(m_{u}+m_{d}\right) / 2}\left(s-\frac{4}{3} m_{\pi}^{2}\right)
$$

where $s=\left(p_{\eta}-p_{\pi^{0}}\right)^{2}$.

In the three flavor case the next to leading Lagrangian contains few additional terms in comparison with (96), (97) 224, 26]

$$
\begin{gathered}
L_{2 e f f}^{\prime}=l_{3} \operatorname{Tr}\left(\partial_{\mu} U \partial_{\mu} U^{+} \partial_{\nu} U \partial_{\nu} U^{+}\right)+l_{5} \operatorname{Tr}\left[\partial_{\mu} U \partial_{\mu} U^{+} \chi\left(U+U^{+}\right)\right]+ \\
+l_{8} \operatorname{Tr}\left(\chi U \chi U^{+}+U \chi^{+} U \chi\right)
\end{gathered}
$$

In the case of three flavours in the order of $p^{4}$, the term of different origin proportional to the totally antisymmetric tensor $\varepsilon_{\mu \nu \lambda \sigma}$ arises. As was pointed out by Wess and Zumino, [39] its occurrence is due to anomalous Ward identities for vector and axial nonsinglet currents. Witten [40] had presented the following heuristic argument in the favor of this term. The leading and next to leading Lagrangians (71), (84), (96), (97), (119) are invariant under discrete symmetries $U(x) \rightarrow U^{+}(x), U(\mathbf{x}, t) \rightarrow U(-\mathbf{x}, t)$. According to (75) this is equivalent to $\varphi_{i}(x) \rightarrow-\varphi_{i}(x)$. In the case of pions this operation coincides with $G$ - parity, but for the octet of pseudoscalar mesons this is not the case. Particularly, such symmetry forbids the process $K^{+} K^{-} \rightarrow \pi^{+} \pi^{-} \pi^{0}$ and $\eta \pi^{0}=\pi^{+} \pi^{-} \pi^{0}$, which are allowed in QCD. In QCD the symmetry under the sign change of pseudoscalar meson fields is valid only if supplemented by space reflection, i.e. $\varphi_{i}(-\mathbf{x}, t) \rightarrow-\varphi_{i}(\mathbf{x}, t)$. Therefore, one may add to chiral lagrangian a term, which is invariant under the latter operation, but violates separately $\mathbf{x} \rightarrow-\mathbf{x}$ and $\varphi_{i}(x) \rightarrow-\varphi_{i}(x)$. Evidently, such term is proportional to $\varepsilon_{\mu \nu \lambda \sigma}$. The general form of the term added to the equation of motion is unique:

$$
\frac{1}{8} f_{\pi}^{2}\left(-\partial_{\mu}^{2} U^{+}+U^{+} \partial_{\mu}^{2} U \cdot U^{+}\right)+\lambda \varepsilon_{\mu \nu \lambda \sigma}\left\{U^{+} \partial_{\mu} U \cdot U^{+} \partial_{\nu} U \cdot U^{+} \partial_{\lambda} U \cdot U^{+} \partial_{\sigma} U\right\}=0,
$$

where $\lambda$ is a constant. (Other nonleading terms are omitted). Eq.(120) is noninvariant under $U^{+} \rightarrow U$ and $\mathbf{x} \rightarrow-\mathbf{x}$ separately, but conserves parity. However, (120) cannot derived from local Lagrangian in four dimensional space-time, because the trace of the second term in the lhs of (120) vanishes. Witten [40] had shown that the Lagrangian can be represented formally as an integral over some five-dimensional manifold, where Lagrangian density is local. The integral over this manifold reduces to its boundary, which is precisely 4-dimensional space-time. In the first nonvanishing order in mesonic fields the contribution to the Lagrangian (the so called Wess-Zumino term [39]) is equal to: $[39-41]$

$$
\Lambda_{W Z}(U)=n \frac{1}{15 \pi^{2} f_{\pi}^{2}} \int d^{4} x \varepsilon_{\mu \nu \lambda \sigma} \operatorname{Tr}\left(\Phi \partial_{\mu} \Phi \partial_{\nu} \Phi \partial_{\lambda} \Phi \partial_{\sigma} \Phi\right)
$$

where $\Phi=\sum \lambda_{m} \varphi_{m}$. The coefficient $n$ in (121) is an integer number [40]. This statement follows from the properties of mapping of 4-dimensional space-time into $S U(3)$ manifold produced by the field $U$. It is clear from (121) that $L_{W Z}=0$ in the case of two flavors: the only antisymmetrical tensor in flavor indices is $\varepsilon^{i k l}$ and it is impossible to construct antisymmetrical in coordinates expression from the derivatives of pionic fields. 
In order to find the value of $n$ it is instructive to consider the interaction with electromagnetic field. In this case the Wess-Zumino Lagrangian is supplemented by terms which form together with (121) a gauge invariant Lagrangian 40

$$
\begin{gathered}
L_{W Z}\left(U, A_{\mu}\right)=L_{W Z}(U)-e n \int d^{4} x A_{\mu} J_{\mu}+\frac{i e^{2} n}{24 \pi^{2}} \int d^{4} x \varepsilon_{\mu \nu \lambda \sigma}\left(\partial_{\mu} A_{\nu}\right) A_{\lambda} \times \\
\times \operatorname{Tr}\left[e_{q}^{2}\left(\partial_{\sigma} U\right) U_{+} e_{q}^{2} U^{+}\left(\partial_{\sigma} U\right)+e_{q} U e_{q} U^{+}\left(\partial_{\sigma} U\right) U^{+}\right],
\end{gathered}
$$

where

$$
\begin{gathered}
J_{\mu}=\frac{1}{48 \pi^{2}} \varepsilon_{\mu \nu \lambda \sigma} \operatorname{Tr}\left[e_{q}\left(\partial_{\nu} U \cdot U^{+}\right)\left(\partial_{\lambda} U \cdot U^{+}\right)\left(\partial_{\sigma} U \cdot U^{+}\right)+\right. \\
\left.+e_{q}\left(U^{+} \partial_{\nu} U\right)\left(U^{+} \partial_{\lambda} U\right)\left(U^{+} \partial_{\sigma} U\right)\right]
\end{gathered}
$$

$e_{q}$ is the matrix of quark charges, $e_{q}=\operatorname{diag}(2 / 3,-1 / 3,-1 / 3)$ and $e$ is the proton charge. The amplitude of $\pi^{0} \rightarrow \gamma \gamma$ decay can be found from the last term in (122). It is given by

$$
T\left(\pi^{0} \rightarrow \gamma \gamma\right)=\frac{n e^{2}}{48 \sqrt{2} \pi^{2} f_{\pi}} \varepsilon_{\mu \nu \lambda \sigma} F_{\mu \nu} F_{\lambda \sigma}
$$

On the other side, the same amplitude is determined in QCD by anomaly. Use the anomaly condition 42 - 44

$$
\partial_{\mu} j_{\mu 5}^{3}=\frac{\alpha}{2 \pi} N_{c}\left(e_{u}^{2}-e_{d}^{2}\right) F_{\mu \nu} \tilde{F}_{\mu \nu}=\frac{\alpha}{12 \pi} N_{c} \varepsilon_{\mu \nu \lambda \sigma} F_{\mu \nu} F_{\lambda \sigma}
$$

where $N_{c}$ is the number of colors and $e_{u}, e_{d}$ are $u$ and $d$ quark charges. For the amplitude $T\left(\pi^{0} \rightarrow \gamma \gamma\right)$ we have, exploiting the PCAC condition (65):

$$
T\left(\pi^{0} \rightarrow \gamma \gamma\right)=\frac{e^{2}}{48 \sqrt{2} \pi^{2} f_{\pi}} N_{c} \varepsilon_{\mu \nu \lambda \sigma} F_{\mu \nu} F_{\lambda \sigma}
$$

(124) coincides with (125), if $n=N_{c}$ 40]. The other physically interesting object, the $\gamma \pi^{+} \pi^{-} \pi^{0}$ vertex is determined by the second term in the rhs of (122) and is equal to

$$
\Gamma\left(\gamma \pi^{+} \pi^{-} \pi^{0}\right)=-\frac{1}{3} i e \frac{n}{\pi^{2} \sqrt{2} f_{\pi}^{3}} \varepsilon_{\mu \nu \lambda \sigma} A_{\mu} \partial_{\nu} \pi^{+} \partial_{\lambda} \pi^{-} \partial_{\sigma} \pi^{0}
$$

Again, if $n=N_{c}$, this result agrees with QCD calculations based on VAAA anomaly or with the phenomenological approach, where the anomaly was taken as granted [45]- 47.

The chiral effective theory is valid also for the pion-baryon low energy interaction, where a lot of results was obtained. We restrict ourselves here to presenting of effective pion-nucleon interaction Lagrangian in the leading order (see e.g.[1], a good review, where high order terms are considered is in [48]):

$$
L_{\pi N}=-\frac{g_{A}}{f_{\pi} \sqrt{2}} \bar{\psi}_{N} \gamma_{\mu} \gamma_{5} \vec{\tau} \partial_{\mu} \vec{\varphi} \psi_{N}-\frac{1}{2 f_{\pi}^{2}} \bar{\psi}_{N} \gamma_{\mu} \vec{\tau}\left[\vec{\varphi} \partial_{\mu} \vec{\varphi}\right] \psi_{N},
$$

where $\psi_{N}$ are nucleon spinors and $g_{A}$ is the axial neutron $\beta$-decay constant, $g_{A}=1.26$. The first term in (128) is a standard pion-nucleon interaction with pseudovector coupling, the second one represents the contact $\pi \pi N \bar{N}$ interaction. 


\section{Low energy sum rules in CET}

Using CET technique important low energy sum rules can be derived, which of course, are valid also in QCD. The most interesting, which are tested by experiment, refer to the difference of the polarization operators of vector and axial currents. Let us define

$$
\begin{gathered}
\Pi_{\mu \nu}^{U}(q)=i \int d^{4} x e^{i q x}\left\langle 0 \mid T\left\{U_{\mu}(x), U_{\nu}(0)^{+}\right\} 0\right\rangle= \\
=\left(q_{\mu} q_{\nu}-q^{2} \delta_{\mu \nu}\right) \Pi_{U}^{(1)}\left(q^{2}\right)+q_{\mu} q_{\nu} \Pi_{U}^{(0)}\left(q^{2}\right)
\end{gathered}
$$

where

$$
U=V, A \quad V_{\mu}=\bar{u} \gamma_{\mu} d, \quad A_{\mu}=\bar{u} \gamma_{\mu} \gamma_{5} d,
$$

$V_{\mu} 4$ and $A_{\mu}$ are vector and axial quark currents. The imaginary parts of the correlators are the so-called spectral functions $\left(s=q^{2}\right)$ :

$$
v_{1}(s) / a_{i}(s)=2 \pi \operatorname{Im} \Pi_{V / A}^{(1)}(s), \quad a_{0}(s)=2 m \operatorname{Im} \Pi_{A}^{(0)}(s),
$$

which are measured in $\tau$-decay. (Isotopically related to $v_{1}$ spectral function is measured in $e^{+} e^{-}$- annihilation). The spin 0 axial spectral function $a_{0}(s)$ which is mainly saturated by one pion state will not be interesting for us now.

$\Pi_{V}^{(1)}(s)$ and $\Pi_{A}^{(1)}(s)$ are analytical functions of $s$ in the complex $s$-plane with a cut along the right semiaxes, starting from the threshold of the lowest hadronic state: $4 m_{\pi}^{2}$ for $\Pi_{V}^{(1)}$ and $9 m_{\pi}^{2}$ for $\Pi_{A}^{(1)}$. Besides the cut, $\Pi_{a}^{(1)}\left(q^{2}\right)$ has a kinematical pole at $q^{2}=0$. This is a specific feature of QCD and CET, which follows from the chiral symmetry in the limit of massless $u, d$-quarks and its spontaneous violation. In this limit axial current is conserved and a massless pion exists. Its contribution to the axial polarization operator is given by

$$
\Pi_{\mu \nu}^{A}(q)_{\pi}=f_{\pi}^{2}\left(\delta_{\mu \nu}-\frac{q_{\mu} q_{\nu}}{q^{2}}\right)
$$

When the quark masses are taken into account, then in the first order of quark masses, or, what it is equivalent, in $m_{\pi}^{2}$ Eq.132 is modified to:

$$
\Pi_{\mu \nu}^{A}(q)_{\pi}=f_{\pi}^{2}\left(\delta_{\mu \nu}-\frac{q_{\mu} q_{\nu}}{q^{2}-m_{\pi}^{2}}\right)
$$

Decompose (133) in the tensor structures of (129)

$$
\Pi_{\mu \nu}^{A}(q)_{\pi}=-\frac{f_{\pi}^{2}}{q^{2}}\left(q_{\mu} q_{\nu}-\delta_{\mu \nu} q^{2}\right)-\frac{m_{\pi}^{2}}{q^{2}} q_{\mu} q_{\nu} \frac{f_{\pi}^{2}}{q^{2}-m_{\pi}^{2}}
$$

The pole in $\Pi_{1}^{A}\left(q^{2}\right)$ at $q^{2}=0$ is evident.

Let us write dispersion relation for $\Pi_{1}^{V}(s)-\Pi_{1}^{A}(s)$. This may be nonsubtracted dispersion relation, since perturbative terms (besides the small contribution from $u, d$ quarks mass square) cancels in the difference, and the OPE terms decrease with $q^{2}=s$ at least as $s^{-2}$ (the term $\sim m_{q}\langle 0|\bar{q} q| 0\rangle$ in OPE). We have 

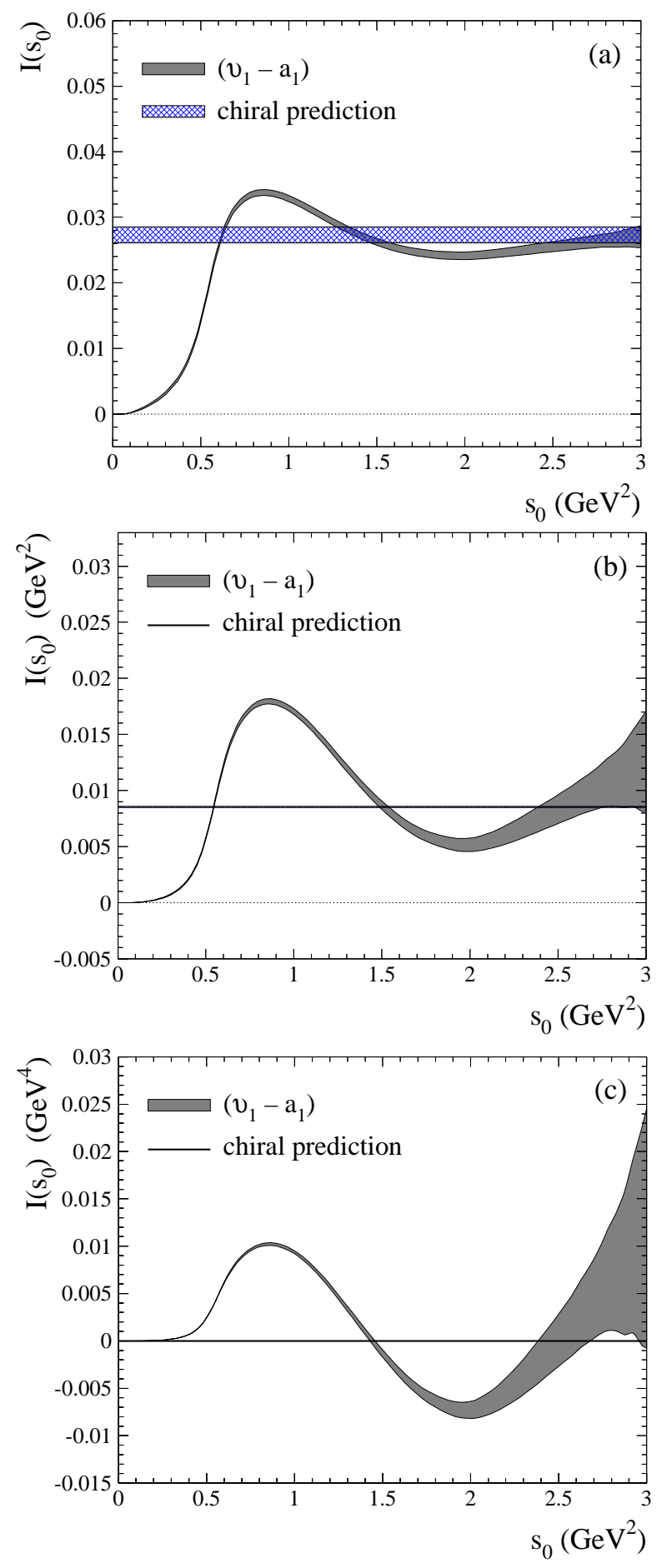

Figure 4a,b,c. The sum rules (136), (137), (138) correspondingly as functions of upper limits of integration $s_{0}$. (Data of ALEPH [52]) 


$$
\Pi_{1}^{V}(s)-\Pi_{1}^{A}(s)=\frac{1}{2 \pi^{2}} \int_{0}^{\infty} d s^{\prime} \frac{v_{1}\left(s^{\prime}\right)-a_{1}\left(s^{\prime}\right)}{s^{\prime}-s}+\frac{f_{\pi}^{2}}{s}
$$

The last term in the rhs of (135) represents the kinematical pole contribution. Let us go to $s \rightarrow \infty$ in (135). Since $\Pi_{1}^{V}(s)-\Pi_{1}^{A}(s) \rightarrow s^{-2}$ in this limit we get the sum rule (the first Weinberg sum rule [49]):

$$
\frac{1}{2 \pi^{2}} \int_{0}^{\infty} d s\left[v_{1}(s)-a_{1}(s)\right]=f_{\pi}^{2}
$$

The accuracy of this sum rule is of order of chiral symmetry violation in QCD, or next order terms in CET, i.e. $\sim m_{\pi}^{2} / M^{2}$ (e.g. a subtraction term).

If the term $\sim m_{q}\langle 0|\bar{q} q| 0\rangle \sim f_{\pi}^{2} m_{\pi}^{2}$ in OPE may be neglected, then, performing in (135) the expansion up to $1 / s^{2}$ we get the second Weinberg sum rule:

$$
\int_{0}^{\infty} s d s\left[v_{1}(s)-a_{1}(s)\right]=O\left(m_{\pi}^{2}\right)
$$

(For other derivations of these sum rules - see [50]).

I present here one more sum rule derived in CET (in its earlier version - PCAC):

Das-Mathur-Okubo sum rule [51]:

$$
\frac{1}{4 \pi^{2}} \int_{0}^{\infty} d s \frac{1}{s}\left[v_{1}(s)-a_{1}(s)\right]=\frac{1}{6} f_{\pi}^{2}\left\langle r_{\pi}^{2}\right\rangle-F_{A},
$$

where $\left\langle r_{\pi}^{2}\right\rangle$ is the mean pion electromagnetic radius and $F_{A}$ is the pion axial vector formfactor in the decay $\pi^{-} \rightarrow m^{-} \nu_{\mu} \gamma$ (in fact, $F_{A}$ is a constant with high accuracy).

The comparison of the sum rules $(135,136,137)$ with the results of the recent measurements of $v_{1}(s)-a_{1}(s)$ in $\tau$-decay by ALEPH collaboration [52] are presented in Fig.4 versus the upper limit of integration.

\section{QCD and CET at finite temperature}

CET is a useful tool for study QCD at finite temperature. It is a common believe, that with temperature increase any hadronic system undergoes a phase transition with restoration of chiral symmetry and liberation of colors - deconfinement (for reviews see [5355]. These two phenomena can proceed in a single phase transition or may be separated. The estimation of the critical temperature(s) $T_{c}$ were found from lattice calculations, from studies of suitable correlation functions, in the framework of models and from the study of temperature dependence of condensate in the framework of CET. All of this indicates that $T_{c} \approx 150-250 \mathrm{MeV}$.

I present here the simple argument [56], based on the consideration on any hadronic correlator $P(x)$ at large space-like distances $\mathbf{x}$. One may expect, that

$$
P(x) \sim e^{-\mu(T)|\mathbf{x}|}
$$


where $\mu(T)$ is temperature depending screening parameter. Eq.139 is valid if: 1) $\mu|\mathbf{x}| \gg$ $1 ; 2)|\mathbf{x}| \lesssim\left(\alpha_{s}(T) T\right)^{-1}$, because at such $|\mathbf{x}|$ the infrared divergence arises in the theory [57]. At high temperature $\mu(T)$ is given by Matsubara frequency

$$
\mu=2 \pi T \text { for bosons (two quarks) }, \quad \mu=3 \pi T \text { for baryons (three quarks) }
$$

At low $T \mu(T)$ is equal by the mass of the corresponding hadron (except for pion, where the conditions 1 and 2 cannot be satisfied simultaneously). Taking, as examples, $\rho$ and $a_{1}$-mesons, we find that the matching of two regimes occurs at $150-200 \mathrm{MeV}$.

Quark condensate may be considered as an order parameter in QCD. Its vanishing at some critical temperature $T=T_{c}$ would indicate on the phase transition - the restoration of chiral symmetry at $T=T_{c}$. Taking this in mind, calculate $T^{2}$ correction to $\langle 0|\bar{u} u|$ $0\rangle=\langle 0|\bar{d} d| 0\rangle$ quark condensate in the limit of massless $u, d$ quarks [58,59].

The mean value of any operator $O$ at finite temperature is given by

$$
\langle O\rangle_{T}=\sum_{n}\left\langle n\left|O \frac{1}{e^{H / T} \pm 1}\right| n\right\rangle \rho_{n},
$$

where \pm signs refer to Fermi and Bose systems, $\rho$ is the density of the state $|n\rangle$. At low $T$ and massless $u, d$ quarks the main contribution comes from states of massless pions. Contributions of all other particles are exponentially suppressed by factors $e^{-m / T}$ where $m$ is the particle mass. (Summation over $n$ should be performed over Hilbert space of physical particles, since at small $T$ the system is in confinement phase and the problem is characterized by large distances). In the order of $T^{2}$ it is enough to account in (140) only one pion state. This gives

$$
\Delta_{T}\langle\bar{u} u\rangle=3 \int \frac{d^{3} p}{(2 \pi)^{3} \cdot 2 E}\left\langle\pi^{+}|\bar{u} u| \pi^{+}\right\rangle \frac{1}{e^{E / T}-1}
$$

where $\Delta_{T}$ means the temperature correction and factor 3 comes from 3 pion states $\pi^{+}, \pi^{-}, \pi^{0}$. It is clear that the one-pion phase space factor results in required power $T^{2}$, two-pion states give $T^{4}$ etc. From QCD Lagrangian we have

$$
\left\langle\pi^{+}|\bar{u} u| \pi^{+}\right\rangle=-\frac{\partial}{\partial m_{u}}\left\langle\pi^{+}|L| \pi^{+}\right\rangle
$$

Substitution of the chiral effective Lagrangian (85) into (143) instead of the QCD Lagrangian leads to

$$
\left\langle\pi^{+}|\bar{u} u| \pi^{+}\right\rangle=\frac{1}{2} B\left\langle\pi^{+}\left|2 \varphi^{+} \varphi\right| \pi^{+}\right\rangle=B=-\frac{2}{f_{\pi}^{2}}\langle 0|\bar{u} u| 0\rangle
$$

Therefore,

$$
\Delta_{T}\langle\bar{u} u\rangle=-\frac{6}{f_{\pi}^{2}}\langle 0|\bar{u} u| 0\rangle \int \frac{d^{3} p}{(2 \pi)^{3} \cdot 2 E} \frac{1}{e^{E / T-1}}=-\frac{T^{2}}{4 f_{\pi}^{2}}\langle 0|\bar{u} u| 0\rangle
$$

Quark condensate decreases with increasing of temperature. If such linear with $T^{2}$ behaviour would continue up to $T=2 f_{\pi}=\simeq 250 \mathrm{MeV}$, quark condensate would vanish at 


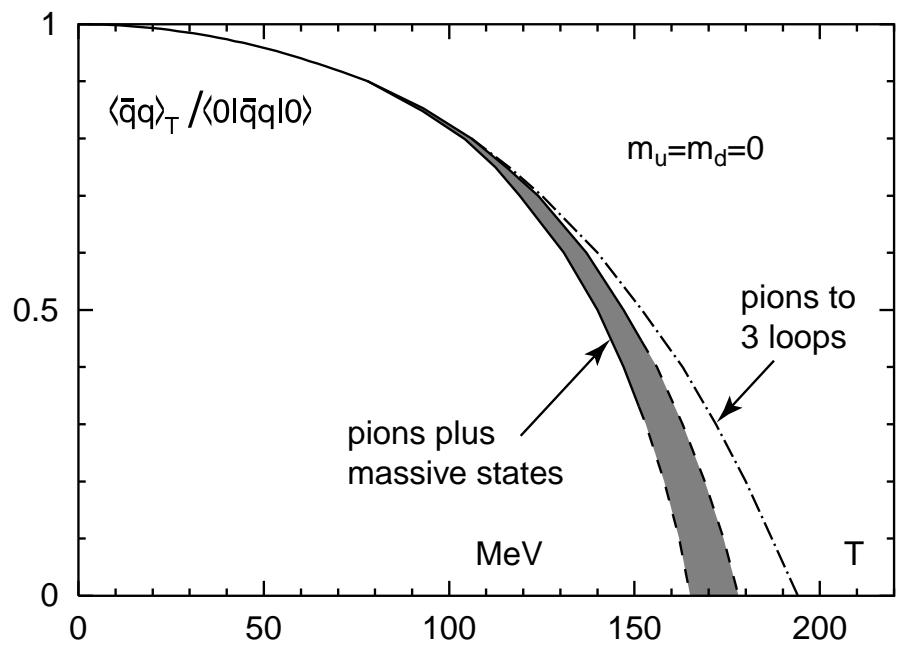

Figure 5. Temperature dependence of quark condensate up to 3 loops (at $m_{u}=m_{d}=0$ ) - Eq.146 - dot-dashed line. The shaded area is the same with model account of massive states (from Ref.61).

this temperature and chiral symmetry would be restored. In fact, the calculation of higher order terms in $T^{2}$ (up to $T^{6}$ ) gives $[60,61]$

$$
\langle\bar{q} q\rangle_{T}=\langle 0|\bar{q} q| 0\rangle\left[1-\frac{N_{f}^{2}-1}{N_{f}} \frac{T^{2}}{6 f_{\pi}^{2}}-\frac{N_{f}^{2}-1}{2 N_{f}^{2}}\left(\frac{T^{2}}{6 f_{\pi}^{2}}\right)^{2}-N_{f}\left(N_{f}^{2}-1\right)\left(\frac{T^{2}}{6 f_{\pi}^{2}}\right)^{3} \cdot \ln \frac{M}{T}\right]
$$

where $N_{f}$ is the number of flavors $\left(N_{f}=2\right.$ for $u, d$ massless quarks) and $M$ is a cutoff. All three terms in the expansion have the same sign what indicates lowering phase transition temperature, up to $T_{c} \sim 150 \mathrm{MeV}$. Quark condensate temperature dependence at low $T$ is shown on Fig.5.

For gluonic condensate the situation is more subtle. The operator $G_{\mu \nu} G_{\mu \nu}$ is proportional to the trace of the energy-momentum tensor $\theta_{\mu \nu}$ and the latter is generator of conform transformation. However, the massless non-interacting pion gas is conformally invariant. (Pions are non-interacting at low $T$ because of the Adler theorem). For this reason the low temperature expansion for gluonic condensate starts from $\sim T^{8}$ term [60].

Consider finally $T^{2}$ corrections to the correlators of vector and axial currents in the limit of massless quarks [62]. At finite $T$ the correlators are defined as $\left(q^{2}=-Q^{2}<0\right)$.

$$
\Pi_{\mu \nu}^{V}(q, T)=i \int d^{4} x e^{i q x} \sum_{n}\left\langle n\left|T V_{\mu}^{a}(x), V_{\nu}^{a}(0) \exp [(\Omega-H) / T]\right| n\right\rangle
$$

where

$$
V=V, A \quad V_{\mu}^{a}=\bar{q} \gamma_{\mu} \frac{\tau^{a}}{2} q, \quad A_{\mu}^{a}=\bar{q} \gamma_{\mu} \gamma_{5} \frac{\tau_{a}}{2} q
$$

and $e^{-\Omega / T}=\sum_{n}\left\langle n\left|e^{-H / T}\right| n\right\rangle$. To evaluate $\Pi_{\mu \nu}^{V}(q, T)$ at low temperature, $T^{2} \ll Q^{2}$ in the sum over $|n\rangle$ in (147) only the vacuum and pion states must be taken into account. The matrix elements 


$$
\left\langle\pi\left|T\left\{U_{\mu}^{a}(x), U_{\nu}^{a}(0)\right\}\right| \pi\right\rangle
$$

are easily evaluated applying reduction formulas to pions and using Eq.(65). Equal time commutators, which arise, are calculated by current algebra relations (or can be derived from Eq.78). The integration over pionic phase space (relativistic Bose gas) can be done with the help of the formula (for massless pions):

$$
\int \frac{d^{3} k}{(2 \pi)^{3}} \frac{1}{2 k} \frac{1}{e^{k / T}-1}=\frac{1}{24} T^{2}
$$

The result is:

$$
\begin{aligned}
& \Pi_{\mu \nu}^{V}(q, T)=(1-\varepsilon) \Pi_{\mu \nu}^{V}(q, 0)+\varepsilon \Pi_{\mu \nu}^{A}(q, 0) \\
& \Pi_{\mu \nu}^{A}(q, T)=(1-\varepsilon) \Pi_{\mu \nu}^{A}(q, 0)+\varepsilon \Pi_{\mu \nu}^{V}(q, 0)
\end{aligned}
$$

where $\varepsilon=T^{2} / 3 f_{\pi}^{2}$. If $\Pi_{\mu \nu}^{V / A}(q, 0)$ are represented through dispersion relations by contributions of physical states in $V$ and $A$ channels, say $\rho, a_{1}, \pi$ etc poles, then according to (151) in the correlators $\Pi^{V, A}(q, T)$ the poles do not shift in order $T^{2}$ and appear at the same position as at $T=0$. The consequence of (151) is that, at $T \neq 0$ in transverse vector channel apart from the poles corresponding to vector particle, there arise poles, corresponding to axial particles and vice verca. In the same way a pion pole appears in the longitudinal part of vector channel. The same phenomenon of parity mixing (and, in some cases also isospin mixing) appears at finite $T$ also in other channels, including baryonic channels [63].

\section{Conclusion}

The goal of this review is to convince the reader, that chiral effective theory (CET) of strong interactions is: on one hand a direct consequence of QCD, of the chiral symmetry of QCD and its spontaneous violation; and on the other hand, a very effective tool with high predictive power for solving the problems of strong interactions at low energies. It was demonstrated, that in QCD the masses of light quarks $(u, d$ and, in some extent, also $s$ ) are small and in a good approximation, when these masses are neglected, QCD is chirally symmetric. However, the physical spectrum of real world (including the vacuum state) does not possesses this symmetry: there is nonvanishing (in the limit $m_{u}, m_{d} \rightarrow 0$ ) symmetry violating quark condensate, and the baryon masses are by no means small, in contradiction with chiral symmetry. It was shown, that these two facts - the large baryon masses and the appearance of quark condensate are tightly interconnected: the first can be expressed through the second. The violation of chiral symmetry on the physical spectrum means that chiral symmetry is broken spontaneously. The direct consequence of this fact is the appearance of massless Goldstone bosons in the spectrum (pion in case of $S U(2)$ symmetry, where $u$ and $d$-quarks are considered as massless and $s$-quark as massive). The known symmetry of the theory and the existence of massless Goldstone bosons allows one to construct CET, valid at low energies. CET is an effective theory, what means, 
that, when going to the next approximation - higher powers of particle momenta - new additional terms in the theory Lagrangian appear.

In the review CET Lagrangian in the first and second orders in momenta was explicitly constructed and its main features were discussed. On few examples it was demonstrated that CET is very powerful in consideration of low energy interactions of pions. Low energy sum rules, which are the subject of direct experimental test, were presented. It was demonstrated, that CET is very suitable tool for the study of QCD at finite temperature. The indications for phase transitions in QCD were obtained from this study.

I am very thankful to H.Leutwyler for enlightening discussion of various aspects of CET, I learned a lot from his papers and reviews on this subject. I am also very indebted to him for his hospitality at Bern.

This work was made possible in part by Award No. RP2-2247 of U.S. Civilian Research and Development Foundation for Independent States of Former Soviet Union (CRDF), by Russian Found of Basic Research grant 00-02-17808, and INTAS Call 2000 Grant (Project $587)$. 


\section{References}

[1] A.I.Vainstein and V.I.Zakharov, Usp.Fiz.Nauk, 100 (1970) 225.

[2] J.Gasser and H.Leutwyler, Nucl. Phys. B94 (1975) 269.

[3] S.Weinberg in: A.Festschrift for I.I.Rabi, ed. L.Motz, Trans. New York Acad. Sci., Ser.II, 38 (1977) 185.

[4] T.Appelquist and J.Carrazone, Phys. Rev. D11 (1975) 2856.

[5] R.Dashen. Phys.Rev. 183 (1969) 1245.

[6] Gasser and H.Leutwyler, Phys. Rep. 87 (1982) 77.

[7] H.Leutwyler, Journ. Moscow Phys. Soc. 6 (1996).

[8] M.Gell-Mann, R.J.Oakes and B.Renner, Phys. Rev. 175 (1968) 2195.

[9] M.L.Goldberger and S.B.Treiman, Phys. Rev. 110 (1958) 1178.

[10] J.Goldstone, Nuovo Cim 19 (1961) 154.

[11] Y.Nambu and G.Jona-Lasinio, Phys.Rev. 122 (1961) 345.

[12] J.Goldstone, A.Salam and S.Weinberg, Phys.Rev. 127 (1962) 965

[13] S.Coleman, in: "Laws of hadronic matter", Proc.of 11 Course of the "Ettore Maiorana" Intern.School of Subnuclear Physics ed. by A.Zichichi Academic Press, London and New York, 1975.

[14] M.A.Shifman, A.I.Vainshten, V.I.Zakharov, Nucl. Phys. B147 (1979) 385, 448.

[15] B.L.Ioffe, Nucl. Phys. B188 (1981) 317, E: B192 (1982) 591.

[16] Vacuum Structure and QCD Sum Rules, Ed.M.Shifman, North Holland, Amsterdam, 1992.

[17] Y.Chung, H.G.Dosch, M.Cremer, D.Schall, Nucl. Phys. B197 (1982) 57.

[18] B.L.Ioffe, Zs. Phys. C18 (1983) 67.

[19] V.M.Belyaev, B.L.Ioffe, ZhETF, 83 (1982) 876.

[20] V.M.Belyaev, B.L.Ioffe, ZhETF, 84 (1983) 1236.

[21] B.L.Ioffe, Acta Physica Polonica B16 (1985) 543.

[22] S.L. Adler, Phys. Rev. 137 (1965) B1022, 139 (1965) B1638.

[23] S.Weinberg, Physica, A96 (1979) 327. 
[24] Leutwyler, Lectures at the XXX Internationale Universitätswochen für Kernphysik, Schladming, Austria, 1991.

[25] J.Gasser and H.Leutwyler, Ann. Phys. (N.Y.) 158 (1984) 142.

[26] J.Gasser and H.Leutwyler, Nucl. Phys. B250 (1985) 465.

[27] S.Coleman, J.Wess and B.Zumino, Phys. Rev. 177 (1969) 2239.

[28] C.G.Callan, Jr., S.Coleman, J.Wess and B.Zumino, Phys. Rev. 177 (1969) 2247.

[29] S.Weinberg, Phys. Rev. Lett. 17 (1966) 616.

[30] P.Langacker and H.Pagels, Phys. Rev. D8 (1973) 4595.

[31] M.A.B.Beg and A.Zepeda, Phys. Rev. D6 (1972) 2912.

[32] M.K.Volkov and V.N.Pervushin, Yad. Fiz. 20 (1974) 762, (Sov. J. Nucl. Phys. 20 (1975) 408.

[33] V.A.Novikov, M.A.Shifman, A.I.Vainstein and V.I.Zakharov, Nucl. Phys. B191 (1981) 301.

[34] B.L.Ioffe, Yad. Fiz. 29 (1979) 1611 (Sov. J. Nucl. Phys. 20 (1979) 827.

[35] D.J.Gross, S.B.Treiman and Wilczek, Phys. Rev. D19 (1979) 2188.

[36] B.L.Ioffe, M.A.Shifman, Phys. Lett. 95B (1980) 99.

[37] H.Osborn and D.R.Wallace, Nucl. Phys. B20 (1970) 23.

[38] J.Gasser and H.Leutwyler, Nucl. Phys. B250 (1985) 539.

[39] J.Wess and B.Zumino, Phys. Lett B37 (1971) 95.

[40] E.Witten, Nucl. Phys. B223 (1983) 422.

[41] I.Zahed and G.E.Brown, Phys. Rep. 142 (1986) 1.

[42] S.L.Adler, Phys. Rev. 1771969147.

[43] J.S.Bell and R.Jackiw, Nuovo Cim 60 (1969) 1517.

[44] W.A.Bardeen Phys. Rev. 184 (1969) 1848.

[45] M.V.Terentjev, Pisma v ZhETF 14 (1971) 140.

[46] S.Adler, B.Lee, S.Treiman and A.Zee, Phys. Rev. D4 (1971) 3497.

[47] M.V.Terentjev, Usp. Fiz. Nauk, 112 (1974) 37.

[48] U.-G.Meissner, Chiral nucleon dynamics, Lectures at the Hampton Studies at Jefferson Lab., preprint KFA(TH)-1997-20, hep-ph/9711365. 
[49] S.Weinberg, Phys. Rev. Lett. 18 (1967) 188, 507.

[50] B.L.Ioffe, V.A.Khoze, L.N.Lipatov, Hard Processes, North Holland, Amsterdam, 1984.

[51] T.Das, V.S.Mathur, S.Okubo, Phys. Rev. Lett. 19859 (1967).

[52] ALEPH Collaboration: R.Barate et al, Eur. J. Phys. C4 409 (1998).

[53] E.V.Shuryak, Phys. Rep. 61 (1980) 71.

[54] D.Gross, R.Pisarski, L.Yaffe, Rev. Mod. Phys. 53 (1981) 43.

[55] McLerran, Rev. Mod. Phys. 58 (1986) 1024.

[56] V.L.Eletsky, B.L.Ioffe, Yad. Fiz. 48 (1988) 602.

[57] A.D.Linde, Rep. Prog. Phys. 42 (1979) 389.

[58] P.Benétruj and M.K.Gaillard, Phys. Rev. D32 (1985) 931.

[59] J.Gasser and H.Leutwyler, Phys. Lett. B184 (1987) 83; B188 (1987) 477.

[60] H.Leutwyler, Nucl. Phys. B (Proc. Suppl.) 4 (1988) 248.

[61] P.Gerber and H.Leutwyler, Nucl. Phys. B321 (1989) 387.

[62] M.Dey, V.L.Eletsky and B.L.Ioffe, Phys. Lett. 252 (1990) 620.

[63] V.L.Eletsky and B.L.Ioffe, Phys. Rev. D47 (1993) 3083. 